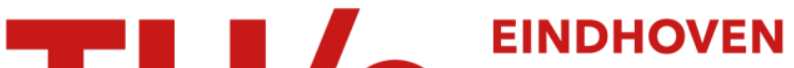

\section{Statistical-learning method for predicting hydrodynamic drag, lift, and pitching torque on spheroidal particles}

\section{Citation for published version (APA):}

Tajfirooz, S., Hausmann, M., Zeegers, J. C. H., Kuerten, J. G. M., \& Fröhlich, J. (2021). Statistical-learning method for predicting hydrodynamic drag, lift, and pitching torque on spheroidal particles. Physical Review E, 103(2), [023304]. https://doi.org/10.1103/PhysRevE.103.023304

DOI:

10.1103/PhysRevE.103.023304

Document status and date:

Published: 15/02/2021

\section{Document Version:}

Publisher's PDF, also known as Version of Record (includes final page, issue and volume numbers)

\section{Please check the document version of this publication:}

- A submitted manuscript is the version of the article upon submission and before peer-review. There can be important differences between the submitted version and the official published version of record. People interested in the research are advised to contact the author for the final version of the publication, or visit the $\mathrm{DOI}$ to the publisher's website.

- The final author version and the galley proof are versions of the publication after peer review.

- The final published version features the final layout of the paper including the volume, issue and page numbers.

Link to publication

\section{General rights}

Copyright and moral rights for the publications made accessible in the public portal are retained by the authors and/or other copyright owners and it is a condition of accessing publications that users recognise and abide by the legal requirements associated with these rights.

- Users may download and print one copy of any publication from the public portal for the purpose of private study or research.

- You may not further distribute the material or use it for any profit-making activity or commercial gain

- You may freely distribute the URL identifying the publication in the public portal.

If the publication is distributed under the terms of Article 25fa of the Dutch Copyright Act, indicated by the "Taverne" license above, please follow below link for the End User Agreement:

www.tue.nl/taverne

Take down policy

If you believe that this document breaches copyright please contact us at:

openaccess@tue.nl

providing details and we will investigate your claim. 


\title{
Statistical-learning method for predicting hydrodynamic drag, lift, and pitching torque on spheroidal particles
}

\author{
S. Tajfirooz $\odot,^{*}$ J. G. Meijer $\odot,^{\dagger}$ and J. G. M. Kuerten $\bullet$ \\ Department of Mechanical Engineering, Eindhoven University of Technology, P.O. Box 513, NL-5600 MB, Eindhoven, The Netherlands \\ M. Hausmann 1 and J. Fröhlich 10 \\ Institute of Fluid Mechanics, Technische Universität Dresden, George-Bähr Strasse 3c, Dresden D-01062, Germany \\ J. C. H. Zeegers \\ Department of Applied Physics, Eindhoven University of Technology, P.O. Box 513, NL-5600 MB, Eindhoven, The Netherlands
}

(Received 22 December 2020; accepted 19 January 2021; published 15 February 2021)

\begin{abstract}
A statistical learning approach is presented to predict the dependency of steady hydrodynamic interactions of thin oblate spheroidal particles on particle orientation and Reynolds number. The conventional empirical correlations that approximate such dependencies are replaced by a neural-network-based correlation which can provide accurate predictions for high-dimensional input spaces occurring in flows with nonspherical particles. By performing resolved simulations of steady uniform flow at $1 \leqslant \operatorname{Re} \leqslant 120$ around a 1:10 spheroidal body, a database consisting of Reynolds number- and orientation-dependent drag, lift, and pitching torque acting on the particle is collected. A multilayer perceptron is trained and validated with the generated database. The performance of the neural network is tested in a point-particle simulation of the buoyancy-driven motion of a 1:10 disk. Our statistical approach outperforms existing empirical correlations in terms of accuracy. The agreement between the numerical results and the experimental observations prove the potential of the method.
\end{abstract}

DOI: 10.1103/PhysRevE.103.023304

\section{INTRODUCTION}

The motion of nonspherical solid particles in viscous fluids has been the subject of research for several decades. Atmospheric particle transport, sediment transport in river beds, material processing and separation, and blood flow are just some examples of flows laden with nonspherical particles. Although the simplifying assumption of a spherical shape is often made, it has been shown that particle nonsphericity can have a significant influence on fluid-particle interactions and, thus, on particle transport [1-4].

Accurate knowledge of hydrodynamics forces and torques acting on particles is of great importance in the numerical study of particle-laden flows. Nonspherical particles are often modeled as fibers, cylinders, or ellipsoids. The smoothness and symmetry properties of spheroids have made them attractive subjects of analytical and numerical studies.

Two general approaches to model fluid-particle interactions in Euler-Lagrange simulations are particle-resolved and point-particle methods. Particle-resolved methods aim at resolving the flow field around the particle and obtaining the hydrodynamic forces and torques through numerical integration of fluid stresses over the surface of the particle. Particle-resolved methods require a grid resolution high

\footnotetext{
*s.tajfirooz@gmail.com

${ }^{\dagger}$ Also at Department of Applied Physics, Eindhoven University of Technology, P.O. Box 513, NL-5600 MB, Eindhoven, the Netherlands.
}

enough to capture the single-particle hydrodynamics. This limits the applicability of such techniques to small fluidparticle systems. Point-particle methods, on the other hand, use empirical or theoretical models for the hydrodynamic interactions between the particle and the fluid. Due to their lower computational costs, point-particle methods have been extensively applied to investigate particle-laden flows [5-8].

The success of point-particle simulations is directly dependent on the accuracy of the incorporated force and torque models. Clift et al. [1] provided a comprehensive review of drag laws for various particle shapes and flow regimes. In the limit of spherical particles, at moderate rotational Reynolds numbers, the coupling between the translational and the rotational motion is small. Analytical and empirical expressions have been derived which describe the hydrodynamic interactions of rigid spherical particles within and beyond the creeping flow limit [9]. Such expressions include several fluidparticle interaction mechanisms, such as steady-state drag, virtual mass, force due to the undisturbed velocity field, and history effects.

The complexity of the motion of nonspherical particles stems from the strong coupling between the translational and the rotational motions at high particle anisotropies. Moreover, unlike for spheres, the nondimensional numbers cannot be based on a single length scale, making the motion of a nonspherical particle dependent on a larger number of influence parameters [1]. In the Stokes regime, analytical force and torque models for nonspherical particles exist. Oberbeck [10] was among the first to investigate the drag force on a low-aspect-ratio spheroidal particle moving along its principal 
axis in a viscous fluid. Jeffery [11] explored the low-Reynolds number $(\mathrm{Re})$ motion of ellipsoidal particles in shear flows and derived expressions for the shear-induced torque on ellipsoids. Under the assumption of creeping (Stokes) flow, Happel and Brenner [12] analytically derived drag and lift coefficients for ellipsoidal particles at different orientations. Their correlation describes the drag coefficient at a given incidence angle by values of the drag coefficient at extrema of the incidence angle and $\sin ^{2}$ function of the incidence angle.

The expressions for force and torque coefficients in creeping flows have been incorporated in Euler-Lagrange simulations of nonspherical particles in laminar and turbulent flows. In such studies it is assumed that the fluid inertial effects are insignificant [4,13-15]. However, it has been observed that such correlations can lead to significant errors at high particle Reynolds numbers [16,17].

Many authors have collected several numerical and experimental data for Reynolds- and orientation-dependent drag, lift, and torque coefficients of nonspherical particles at finite Reynolds numbers [18-30].

In 2008, Loth [21] collected the existing correlations for regular and irregular shapes in the Stokes and Newton regimes and addressed the applicability of combined correlations for intermediate regimes. He found that the sphericity cannot be used as the only input parameter for the prediction of drag force outside the Stokes regime and that these correlations lose their accuracy as deviations from a sphere become large. Later Hölzer and Sommerfeld proposed new correlations based on a broad set of numerical and experimental data that involve different projected areas to account for particle orientation [22,23]. Zastawny et al. [24] used the immersed boundary method (IBM) to investigate different nonspherical particles at different incident angles and Reynolds numbers in the steady flow regime. Based on the work of Rosendahl [20], Zastawny et al. provided shape-specific correlations for drag, lift, and torque on two ellipsoidal particles and a fiber-shaped particle. Due to the limitations of the IBM method, Zastawny et al. [24] used relatively small computational domains for simulations at low Reynolds numbers ( $\operatorname{Re} \leqslant 1$ ), which led to a considerable reduction in the accuracy of their correlations in this regime [27,29].

Jiang et al. [25] numerically studied a 6:1 prolate spheroid at a $45^{\circ}$ incidence angle and compared their results with the correlation of Zastawny et al. for a fiber with the same aspect ratio and that of Hölzer and Sommerfeld [23], obtaining very good agreement at low Reynolds numbers. At higher Reynolds numbers deviations up to $29 \%$ were observed. More recently, Sanjeevi et al. [29] performed extensive lattice Boltzmann simulations of the flow around a 2:5 oblate ellipsoid, a 5:2 prolate ellipsoid, and a 4:1 fiber up to $\operatorname{Re}=2000$ to extract force and torque coefficients and derived separate correlations for these three shapes. Due to the wide range of Reynolds numbers considered by these authors, they were not able to fit their computed data to the original correlations of Zastawny et al. They instead used a modified version of the $\sin ^{2}$ type correlation. Sanjeevi et al. showed that for prolate spheroids of aspect ratio up to 32 , the $\sin ^{2}$ behavior of the drag coefficient derived by Happel and Brenner, can be extended to the high-Reynolds number regime. They also observed that due to the stronger wake contribution to the drag force, the drag coefficient of low-aspect-ratio oblate spheroids does not follow the $\sin ^{2}$ law.

Andersson and Jiang [31] investigated the flow around an inclined 1:6 oblate ellipsoid at low but finite Reynolds numbers and addressed the challenges in simulating flow over bluff bodies at small Reynolds numbers. Their findings also questioned the reliability of finite Reynolds number correlations at Reynolds numbers on the order of 1 .

The shape-specific force and torque correlations can serve as useful models for point-particle simulations of nonspherical particles. However, it has been shown that the applicability of a correlation to other particle shapes is limited. Furthermore, force or torque correlations for nonspherical particles that cover a broad range of particle Reynolds numbers or different particle shapes are scarce. A single correlation is not capable of predicting hydrodynamic loads over a wide range of Reynolds numbers, aspect ratios, and incidence angles. Different correlations are commonly considered for multiple distinct ranges of one or more input parameter(s). The main reason for this is the difficulty in finding a proper fitting approach that considers the large input space in the right way and does not introduce unsatisfactory high biases. Moreover, phenomena, such as indirect particle-particle interactions when particles are close to each other [32], wall effects on particles in the vicinity of a solid wall [1,33], or inertial shear-induced effects in nonuniform particle-laden flows [34] cannot be straightforwardly described using conventional empirical methods.

In this paper, we propose a different approach for predicting the hydrodynamic interactions between nonspherical particles and surrounding fluid. Instead of the conventional curve fitting approach, we use an artificial neural network (NN) to correlate the force and torque data generated by resolved numerical simulations to a set of input parameters. Artificial neural networks have been applied to various areas from data classification and image processing to motion prediction and pattern recognition. Recently, statistical learning has also been used in fluid dynamics and multiphase flows [35]. For example, Sarghini et al. [36] applied a neural network to predict the Smagorinsky constant in large-eddy simulations, Ling et al. [37] derived a neural network-based Reynolds stress closure for Reynolds averaged Navier-Stokes equations. He and Tafti [38] used a neural network to predict the effect of particle volume fraction and distance to neighboring cells on drag force on spheres. Lui and Wolf [39] combined NN-based regression with flow modal decomposition to construct reduced order models for fluid flow over bluff bodies, and Buzzicotti et al. [40] studied the application of convolutional neural networks to the reconstruction of fluid turbulence data.

Neural networks, being stochastic tools, are generally difficult to reproduce. Still, the significant advantage of a neural network is its theoretical ability to approximate every Borel measurable input-output relation [41]. However, in the context of prediction of hydrodynamic interactions, NN-based trend prediction has three distinct advantages over conventional curve-fitting approaches.

First, to obtain a conventional curve-fitting-based correlation from simulation data, a functional approach is necessary. The choice of a fitting analytical function (linear, quadratic, 
$\sin ^{2}$, etc.) can oversimplify an actual complex dependency which might not be captured due to a low number density of data points, inaccuracy of the computational model, or a limited range of data. The forced behavior of the considered function can lead to high deviations, especially for values lying between or outside the fitting points where interpolation or extrapolation is required. If trained properly, a neural network can predict any dependency without a prior assumption, and this way, the difficult task of finding an appropriate fitting function is circumvented.

Second, fitting an analytical function to a partially erroneous dataset can deteriorate the quality of the prediction over a broader input domain. Correlations inherit the errors in the data as intrinsic biases. Such biases generated by conventional curve-fitting approaches can lead to large temporally accumulated errors in results of time-dependent point-particle simulations incorporating such models. In contrast, the variance error typically generated by deep neural network (DNN) predictions is averaged out in time and, therefore, leads to smaller accumulated errors.

Third, several authors have used different computational methods to derive correlations for hydrodynamic forces and torques. Due to the same reasons mentioned above, for a given problem, the results are usually not identical. This leads to a variety of functional approaches for the same relation. The results of the simulations from different authors can not be used because they are incompatible with the respective correlation approaches. Thus, a large number of valuable data cannot be used. A neural network, instead, can process the data of all authors and minimize the effect of the individual errors of every data set.

A few studies have addressed the steady axisymmetric flow over thin oblate ellipsoids [1,18,42-44]. But, orientation- and Reynolds-number-dependent force or torque correlations for low-aspect-ratio spheroids or disks are explored here. We take the incidence angle and the particle Reynolds number as input parameters and employ a statistical approach to predict the coefficients of drag, lift, and pitching torque of a 1:10 oblate spheroid.

The considered range of Reynolds numbers in this paper is $1 \leqslant \mathrm{Re} \leqslant 120$. It is known that in the buoyancy-driven motion of a particle within this regime, the particle has a vertical trajectory and exhibits no appreciable secondary motion [45]. This allows to extract hydrodynamic loads from steadystate body-fixed simulations. We perform a set of resolved simulations to obtain force and torque data. A well-designed feedforward $\mathrm{NN}$ is then trained with a subset of the collected data. We show that the NN-based predictions are, at least, as accurate as predictions of empirical and theoretical correlations. We test the performance of our model by incorporating it in a point-particle simulation of the buoyancy-driven motion of an oblate spheroid in a liquid with a nonlinear hydrostatic pressure gradient.

The mathematical model and the employed numerical method for the resolved simulations are addressed in Sec. II. The data acquisition procedure is discussed in Sec. III. In Sec. IV the results are presented and discussed, the considered feedforward network and the training and validation procedures are addressed, and the performance of the new model

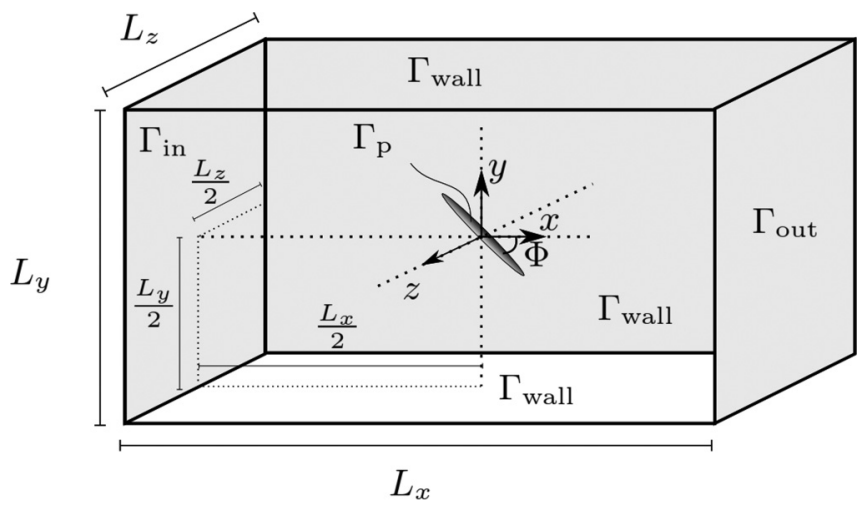

FIG. 1. Sketch of the computational domain $\Omega_{\mathrm{f}}$ and the particle mounted inside the domain.

is tested in a point-particle simulation of a particle settling in a magnetic liquid. Finally, the concluding remarks and future directions are presented in Sec. V.

\section{RESOLVED SIMULATIONS}

\section{A. Mathematical description of the flow}

We solve the flow around a spheroidal particle with length $b$ along the axis of symmetry and maximum diameter normal to this axis equal to $a$. The aspect ratio is defined as $w=b / a$ and fixed to $w=0.1$ in the present case to yield an oblate spheroid. The particle is located in the center of the coordinate system with $x$ as the streamwise, $y$ as the pitchwise, and $z$ as the spanwise coordinate. Indeed, the particle is mounted symmetrically to the $x-y$ plane, and $\Phi$ denotes the pitching angle, i.e., the angle of incidence to the flow. The steady Navier-Stokes equations are solved in a cubic computational domain as shown in Fig. 1. The fluid motion is described by the system,

$$
\begin{aligned}
\nabla \cdot(\mathbf{u} \otimes \mathbf{u}) & =\frac{1}{\rho_{\mathrm{f}}} \nabla \cdot \boldsymbol{\tau} \text { in } \Omega_{\mathrm{f}}, \\
\nabla \cdot \mathbf{u} & =0 \text { in } \Omega_{\mathrm{f}},
\end{aligned}
$$

where the hydrodynamic stress tensor is

$$
\boldsymbol{\tau}=-p \mathbb{I}+\mu_{\mathrm{f}}\left[\nabla \mathbf{u}+(\nabla \mathbf{u})^{\mathrm{T}}\right] .
$$

Here, $\mathbf{u}, p$, and $\rho_{\mathrm{f}}$ are the fluid velocity, the pressure, and the fluid mass density, respectively. The dimensions of the computational domain in streamwise and the two spanwise directions are $L_{x}$ and $L_{z}=L_{y}$. The distance of the particle center from the inlet and the spanwise distances from the walls are $L_{x} / 2$ and $L_{z} / 2=L_{y} / 2$, respectively.

At the inlet boundary $\Gamma_{\text {in }}$, a uniform Dirichlet boundary condition is applied that sets the velocity vector to $\mathbf{u}=$ $\left(u_{0}, 0,0\right)^{\mathrm{T}}$ with $u_{0}=1 \mathrm{~m} / \mathrm{s}$. At the outlet boundary $\Gamma_{\text {out }}$, the stress on the outlet boundary is set to zero. In the case of a backflow, the outlet pressure is adjusted to prevent fluid from entering the domain through the outlet boundary [46]. A slip condition with zero normal velocity and zero tangential shear stress is imposed at the side boundaries $\Gamma_{\text {wall }}$, where

$$
\mathbf{u} \cdot \mathbf{n}=0,\left\{-p \mathbb{I}+\mu\left[\nabla \mathbf{u}+(\nabla \mathbf{u})^{\mathrm{T}}\right]\right\} \mathbf{n}=\mathbf{0} .
$$


At the surface of the spheroid $\Gamma_{\mathrm{p}}$, a no-slip boundary condition is imposed, i.e., $\mathbf{u}=(0,0,0)^{\mathrm{T}}$.

The equatorial diameter of the spheroid $d=2 a$ is taken as the length scale for evaluation of the particle Reynolds number $\operatorname{Re}=\frac{u_{0} \rho_{\mathrm{f}} d}{\mu_{\mathrm{f}}}$. Different Reynolds numbers are achieved by varying the fluids dynamic viscosity $\mu_{\mathrm{f}}$. The particle incidence angle $\Phi$ is changed by rotating the spheroid around the $z$ axis. Forces and torques acting on the particle are obtained by integration of the total stress and its moment around the surface of the particle,

$$
\begin{gathered}
\boldsymbol{F}=\int_{\Gamma_{\mathrm{p}}} \boldsymbol{\tau} \cdot \boldsymbol{n} d A, \\
\boldsymbol{T}=\int_{\Gamma_{\mathrm{p}}} \boldsymbol{r} \times \boldsymbol{\tau} \cdot \boldsymbol{n} d A,
\end{gathered}
$$

with $\boldsymbol{r}$ the distance vector from the center of mass of the particle to a point on the surface $\Gamma_{\mathrm{p}}$.

\section{B. Numerical discretization}

The stabilized Galerkin finite element method in COMSOL MULTIPHYSICS is used to solve (1) and (2) in the weak form. Linear basis functions $(P 1 P 1)$ are used for the velocity and pressure approximations. To ensure numerical stability, the finite element formulation is stabilized by streamline diffusion and crosswind diffusion methods [46]. The weak formulation of (1) is linearized by a "damped" Newton method. The system of linear equations resulting from discretization is solved using the generalized minimal residual method [46].

The surface of the spheroid is discretized using triangular surface elements with a maximum size of $\delta_{\max }$. The volume mesh consists of a combination of tetrahedra and prisms. To accurately capture the boundary layer near the surface of the spheroid and the downstream wake, a swept mesh is considered within the spherical region $x^{2}+y^{2}+z^{2} \leqslant r_{\mathrm{s}}^{2}$, where $r_{\mathrm{s}}$ denotes the radius of the swept region. This swept region is discretized by $n_{\mathrm{s}}$ layers of elements. Their maximum-tominimum element size in the radial direction is $\gamma_{\mathrm{s}}$. Using a swept region which is fixed to the spheroid keeps the mesh skewness at different incidence angles constant. An example of the mesh is shown in Fig. 2.

\section{DATA ACQUISITION}

A database is collected from 266 simulations performed at 14 different Reynolds numbers and 19 different angles of incidence. The two independent input variables are the Re and the angle of incidence $\Phi$. The output parameters are the coefficients of drag, lift, and pitching torque defined as

$$
\begin{gathered}
c_{\mathrm{D}}=\frac{\left|F_{\mathrm{D}}\right|}{\frac{1}{2} \rho_{\mathrm{f}} u_{0}^{2} A}, \\
c_{\mathrm{L}}=\frac{\left|F_{\mathrm{L}}\right|}{\frac{1}{2} \rho_{\mathrm{f}} u_{0}^{2} A}, \\
c_{\mathrm{T}}=\frac{\left|T_{\mathrm{p}}\right|}{\frac{1}{4} \rho_{\mathrm{f}} u_{0}^{2} A d},
\end{gathered}
$$

respectively, where $A=\pi d^{2} / 4$ is the cross sectional area of the spheroid and $F_{\mathrm{D}}=\boldsymbol{F} \cdot \boldsymbol{i}, F_{\mathrm{L}}=\boldsymbol{F} \cdot \boldsymbol{j}$, and $T_{\mathrm{p}}=\boldsymbol{T} \cdot \boldsymbol{k}$ with $\boldsymbol{i}, \boldsymbol{j}$, and $\boldsymbol{k}$ denoting the unit vector in the $x, y$, and $z$

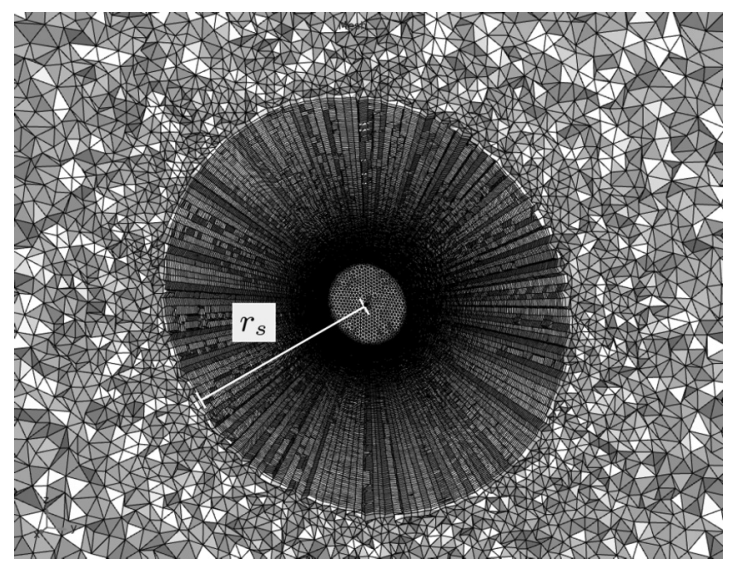

FIG. 2. Example of the mesh in the region close to the spheroid. To accurately resolve the flow in the vicinity of the particle, a spherical swept region with radius $r_{\mathrm{s}}$ is considered around the particle.

directions, respectively. Observe that since the upstream flow is uniform and the computational domain is symmetric in the $z$ direction, the total torque acting on the particle has only one contribution, which is the pitching torque.

The parametric study of Chrust et al. [47] on wake transition scenarios of oblate spheroids at $\Phi=90^{\circ}$ showed that the transition behavior for a thin oblate spheroid is very similar to that of a disk $(w=0)$. The authors observed a similar sevenstage transition scenario in the wake of thin oblate spheroids as for disks. In the thin-disk-like scenario, for a given aspect ratio as the Reynolds number increases from zero, the first (primary) bifurcation at the first critical Reynolds number $\operatorname{Re}_{c, 1}$ leads to a steady nonaxisymmetric wake. At this state, i.e., $\operatorname{Re}_{\mathrm{c}, 1} \leqslant \operatorname{Re} \leqslant \operatorname{Re}_{\mathrm{c}, 2}$, the planar symmetry remains sustained and a steady lift force acts in the wake symmetry plane. As the Reynolds number further increases to the second critical Reynolds number $\mathrm{Re}_{\mathrm{c}, 2}$, the wake transits to a periodic state under a Hopf-type bifurcation, and the planar symmetry vanishes.

A decrease in the aspect ratio of an oblate spheroid leads to an increase in both the first and the second critical Reynolds numbers. For a disk $(w=0)$ Chrust et al. [47] found the Reynolds number range of $117 \leqslant \operatorname{Re} \leqslant 125.2$ for the stability interval of the steady nonaxisymmetric state with planar symmetry. For a 1:6 spheroid, this range was found to be $130 \leqslant$ $\mathrm{Re} \leqslant 137.2$. By interpolating the values for a disk and a 1:6 spheroid, Chrust et al. estimated the first critical Reynolds number to be $\operatorname{Re}_{\mathrm{c}, 1} \approx 130$ for the steady planar-symmetric wake of a 1:10 spheroid. Shenoy and Kleinstreuer [48] observed a value of $\operatorname{Re}_{c, 1}=135$ for the first critical Reynolds number of a 1:10 circular disk. Based on these observations, the particle wake is expected to remain steady at all incidence angles within the considered range of Reynolds numbers in this paper $(\operatorname{Re} \leqslant 120)$. This legitimates conducting steadystate simulations.

Figure 3 illustrates the two-dimensional space of input parameters. Due to the symmetry of the considered geometry, it suffices to consider the incidence angle in the range of $0^{\circ} \leqslant \Phi \leqslant 90^{\circ}$. The number density of data points at lower Reynolds numbers is increased to ensure that the observed 


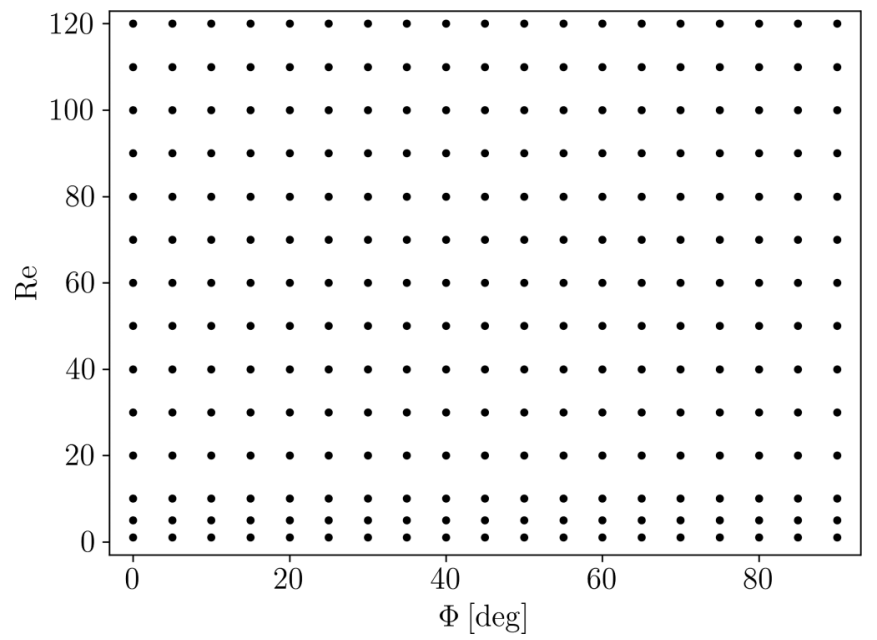

FIG. 3. An illustration of the $\operatorname{Re}-\Phi$ space on which the resolved simulations are performed. Each dot represents one simulation.

strong input-output dependency in this region mentioned by Clift $e$ t al. [1] is accurately captured.

It is well known that the drag, lift, and pitching torque coefficients in the Stokes regime depend linearly on $\mathrm{Re}^{-1}[1]$. To reduce the nonlinearity of the input-output relation, we choose the output variables to be coefficients of drag, lift, and pitching torque multiplied by the Reynolds number. This way, the dependency to be predicted reduces to a constant function in the Stokes regime.

\section{RESULTS AND DISCUSSIONS}

\section{A. Validation of resolved simulations}

First, several simulations have been performed to investigate the sensitivity of the numerical results to different simulation parameters. In particular, the effects of the domain size and the grid resolution on the force and torque coefficients have been studied.

Resolving the flow field at $\Phi=90^{\circ}$ requires the highest spatial resolution [29]. To ensure that the computational grid can capture all the required details of the flow field, we performed the grid sensitivity analysis at this incidence angle. Furthermore, it is known that the effect of domain size becomes more important when the Reynolds number is decreased [18,29,31]. To ensure that confinement effects do not influence the numerical results at low Reynolds numbers, we consider a computational domain with $L=80 \mathrm{~d}$ for cases with $\operatorname{Re}<20$. For cases with higher Reynolds numbers, a domain size of $L=40 d$ is used. This choice is based on the findings of Andersson and Jiang [31].

We validate the numerical results by comparing the results of the present paper with literature data for thin oblate spheroids and disks at $\Phi=90^{\circ}$. As mentioned earlier, the number of studies on thin oblate spheroids is limited. Masliyah and Epstein [42] and Pitter et al. [18] performed axisymmetric simulations to investigate the steady axial flow around spheroids with $0.05 \leqslant w \leqslant 5$ for Reynolds numbers up to $\operatorname{Re}=100$. Within this range of Reynolds numbers, the assumption of an axisymmetric wake is valid even for infinitely thin disks [47]. Based on the results of Pitter et al.
TABLE I. Parameter values of the computational domains and grids.

\begin{tabular}{lcccccc}
\hline \hline Case & $L$ & $\delta_{\max }$ & $r_{\mathrm{s}}$ & $n_{\mathrm{s}}$ & $\gamma_{\mathrm{s}}$ & $n_{\mathrm{e}, \mathrm{t}}$ \\
\hline $\operatorname{Re}<20$ & $80 d$ & $0.02 d$ & $4 d$ & 100 & 80 & $2.31 \times 10^{6}$ \\
$\operatorname{Re} \geqslant 20$ & $40 d$ & $0.02 d$ & $4 d$ & 100 & 160 & $3.68 \times 10^{6}$ \\
\hline \hline
\end{tabular}

[18], Clift et al. [1] suggested the following correlations for the steady drag coefficient of disks and low-aspect ratio oblate spheroids $(w \leqslant 0.05)$ in the range of $0.01<\operatorname{Re} \leqslant 133$,

$$
C_{\mathrm{D}}= \begin{cases}\frac{64}{\pi \mathrm{Re}}\left[1+10^{x}\right], & \text { if } 0.01<\operatorname{Re} \leqslant 1.5, \\ \frac{64}{\pi \operatorname{Re}}\left[1+0.138 \mathrm{Re}^{0.792}\right], & \text { if } 1.5<\operatorname{Re} \leqslant 133,\end{cases}
$$

where $x=-0.883+0.906 \log _{10} \operatorname{Re}-0.025\left(\log _{10} \operatorname{Re}\right)^{2}$. For lower Re, the expression of Oseen can be used,

$$
C_{\mathrm{D}}=\frac{64}{\pi \operatorname{Re}}\left[1+\frac{\operatorname{Re}}{2 \pi}\right] \quad \text { if } \operatorname{Re} \leqslant 0.01 .
$$

In 2006, O'Donnell and Helenbrook [43] performed axisymmetric finite element simulations to extract the drag coefficient of crosswind oblate spheroids within the aspect ratio range $0.1 \leqslant w \leqslant 1$ at $0 \leqslant \operatorname{Re} \leqslant 200$ and derived aspectratio-dependent correlations for the drag coefficient. The drag coefficients obtained from the correlation of O'Donnell and Helenbrook [43] for disks are within $2.7 \%$ of drag coefficients predicted by Pitter et al. [18].

To compare the results of different authors, we define the normalized deviation from the drag coefficient of a sphere with the same cross-sectional area as $\delta_{C_{\mathrm{D}}}=1-\frac{C_{\mathrm{D}, w}}{C_{\mathrm{D}, 1}}$, where $C_{\mathrm{D}, w}$ is the drag coefficient of an oblate spheroid of aspect ratio $w$, and $C_{\mathrm{D}, 1}$ is the drag coefficient of a sphere. The latter is given by [1]

$$
C_{\mathrm{D}, 1}=\left\{\begin{array}{l}
\frac{24}{\operatorname{Re}}\left[1+0.1315 \mathrm{Re}^{a}\right], \\
\frac{24}{\operatorname{Re}}\left[1+0.1935 \mathrm{Re}^{0.6305}\right], \quad \text { if } 0<\operatorname{Re} \leqslant 20,
\end{array}\right.
$$

with $a=0.82-0.05 \log _{10}$ Re. Consecutive grid refinements are performed up to the point where the maximum drag coefficient deviation from the results of O'Donnell and Helenbrook [43] is below $2 \%$. Table I summarizes the chosen computational settings of the simulations.

Figure 4 compares the dependency of $\delta_{C_{\mathrm{D}}}$ on the Reynolds number for spheroids with $w \leqslant 0.1$ based on correlations from literature [1,43], and the results of our simulations in the range of $1 \leqslant \operatorname{Re} \leqslant 120$. Note that the Reynolds number at which the drag coefficient of a sphere equals that of a thin oblate spheroid is $\operatorname{Re} \approx 37$. As the Reynolds number increases the relative difference between the drag coefficient of a disk and that of a 1:10 spheroid increases. The lumped force and torque coefficients collected from the resolved simulations can be found in Appendix.

\section{B. Aspect-ratio-specific features}

Next, we illustrate the effect of the aspect ratio on the incidence-angle and Reynolds-number dependency of drag, lift, and torque coefficients of oblate spheroids. The results 


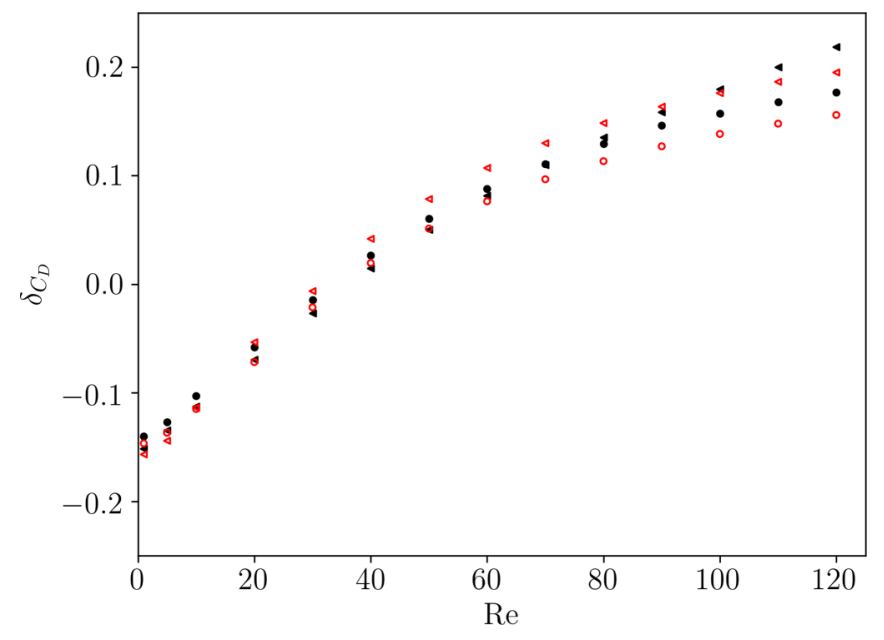

FIG. 4. Deviations of drag coefficients of a 1:10 spheroid and a thin disk at $\Phi=90^{\circ}$ from the drag coefficient of a sphere. The results of the present paper for a 1:10 spheroid [solid bullet $(w=1)$ ] are compared with the correlation of O'Donnell and Helenbrook [43] for a 1:10 spheroid [(open red bullet); $w=0.1$ and (open small left red triangle); $w=0$ ] and correlation of Clift et al. [1] for thin disks [(solid small left triangle) $(w \leqslant 0.05)]$.

of our resolved simulations for a 1:10 spheroid are compared with existing correlations for 1:5 and 2:5 spheroids. For the latter two aspect ratios, we use correlations of Zastawny et al. [24] and Sanjeevi et al. [29], respectively. In our comparison, we keep the particle volume constant and, therefore, define a Reynolds number based on the diameter of a volumeequivalent sphere as $\operatorname{Re}_{\mathrm{e}}=\frac{U_{0} d_{\mathrm{e}}}{v_{\mathrm{f}}}$, where $d_{\mathrm{e}}=w^{1 / 3} d$ denotes the diameter of a sphere with the same volume, and $v_{\mathrm{f}}$ is the fluid kinematic viscosity. The volume-equivalent sphere Reynolds number $\mathrm{Re}_{\mathrm{e}}$ is related to the Reynolds number based on the equatorial diameter through $\operatorname{Re}_{\mathrm{e}}=w^{1 / 3} \mathrm{Re}$. The obtained equivalent drag coefficient based on the cross-sectional area of the volume-equivalent sphere is $c_{\mathrm{D}, \mathrm{e}}=c_{\mathrm{D}} w^{-2 / 3}$. The same relation holds for the coefficients of lift and pitching torques.

\section{Incidence-angle dependency}

In Fig. 5 the incidence-angle dependency of the drag coefficient is compared for the three different aspect ratios. We observe that at a fixed Reynolds number within the considered range, regardless of the particle orientation, a 1:10 spheroid always has a larger drag coefficient compared to $1: 5$ and 2:5 spheroids. The contributions of viscous stress and pressure to the drag force on the 1:10 spheroid are compared in Fig. 6. At low incidence angles, the viscous drag is the dominant contributor to the total drag force. As the incidence angle increases, the contribution of pressure increases, and at sufficiently high incidence angles pressure force has the main contribution to the total drag force. The incidence angle at which the viscous and pressure drags are equal decreases from approximately $\Phi \approx 40$ to $\Phi \approx 20$ by increasing the Reynolds number from $\mathrm{Re}_{\mathrm{e}}=0.5$ to $\mathrm{Re}_{\mathrm{e}}=55.7$. The larger surface area of a 1:10 spheroid compared to the one of 1:5 and 2:5 spheroids leads to a higher viscous drag at low incidence angles. At higher incidence angles the larger recirculation region behind the 1:10 spheroid leads to a more significant pressure drop and, therefore, a relatively larger pressure drag resulting in a larger total drag force.

Another issue which distinguishes the behavior of drag coefficient for the 1:10 spheroid from that of the high-aspectratio spheroids is the maximum value of the drag coefficient at $\mathrm{Re}_{\mathrm{e}}=55.7$. For a 1:10 spheroid, the maximum drag coefficient occurs at $\Phi \approx 80^{\circ}$. This yields two local maxima for the drag coefficient during a $180^{\circ}$ particle rotation, one at $\Phi \approx 80^{\circ}$, and one at $\Phi \approx 110^{\circ}$. This behavior which is also observed for $1: 4$ and 2:5 spheroids at $\operatorname{Re}_{\mathrm{e}}=100[28,29]$ is not observed for the higher-aspect-ratio spheroids.

The effect of aspect ratio on lift and pitching torque coefficients is illustrated in Figs. 7 and 8, respectively. Both coefficients increase with decreasing the aspect ratio. It can be observed that although at low Reynolds numbers $\left(\operatorname{Re}_{1}=1\right)$ the lift coefficient of a 1:10 spheroid behaves similarly to those of 1:5 and 2:5 spheroids, at larger Reynolds numbers the incidence-angle dependency of the lift coefficient of a 1:10 spheroid significantly deviates from those of larger aspect ratio spheroids. At $\mathrm{Re}_{\mathrm{e}} \approx 46$ the lift coefficient profile for a $1: 10$ spheroid is asymmetric with respect to $\Phi=45^{\circ}$. This is due to the fact that the onset of symmetry breaking of the wake occurs at high incidence angles for the 1:10 spheroid when the Reynolds number is lower. In contrast, for 1:5 and 2:5 spheroids, the symmetry seems to be sustained for up to $\operatorname{Re}_{\mathrm{e}}=55$.7. A similar behavior is observed for the pitching torque coefficient in Fig. 8.

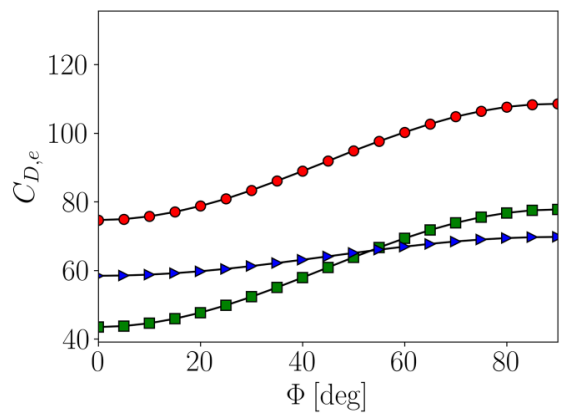

(a)

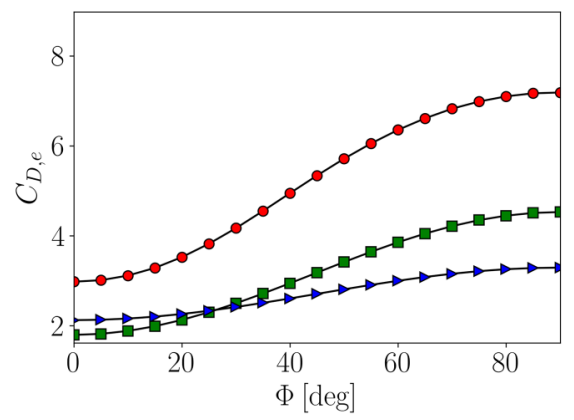

(b)

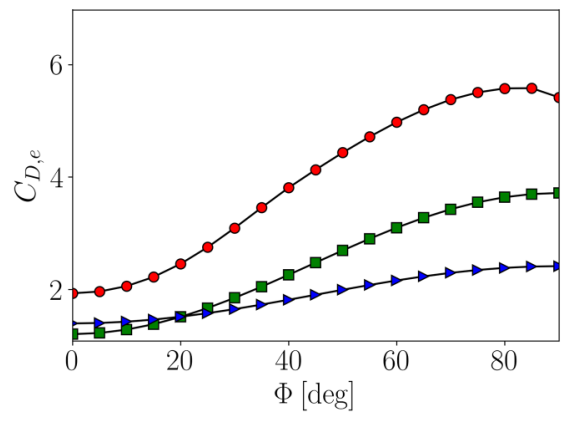

(c)

FIG. 5. Drag coefficient as a function of incidence angle at (a) $\operatorname{Re}_{\mathrm{e}}=0.5\left(\operatorname{Re}_{1}=1\right)$, (b) $\operatorname{Re}_{\mathrm{e}}=27.8\left(\operatorname{Re}_{1}=60\right)$, and (c) $\operatorname{Re}_{\mathrm{e}}=$ $55.7\left(\operatorname{Re}_{1}=120\right)$ [(red solid bullet); $w_{1}=1 / 10$ : (green solid square); $w_{2}=1 / 5$ : and (solid small right triangle); $\left.w_{3}=2 / 5\right]$. 


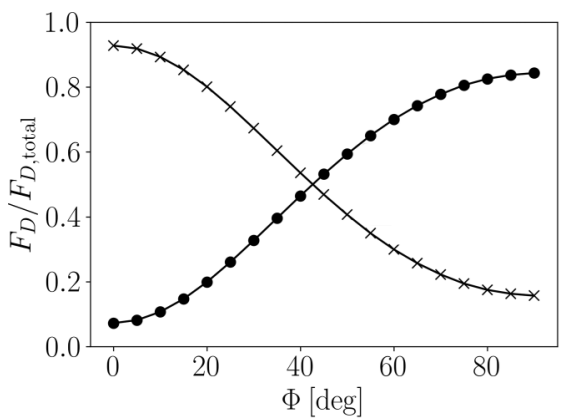

(a)

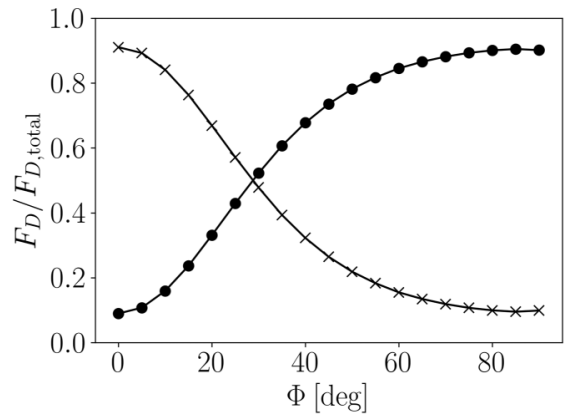

(b)

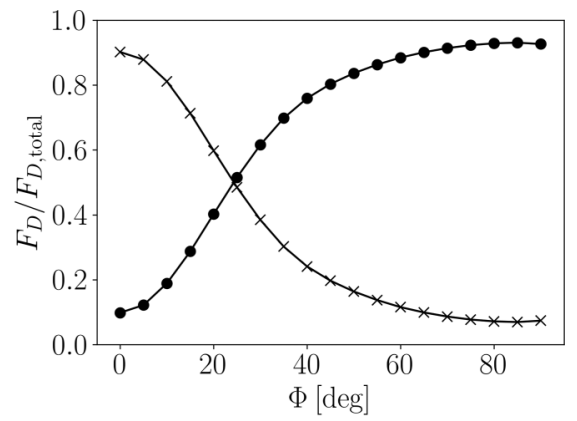

(c)

FIG. 6. Contributions of pressure and viscous stress to the drag coefficient at (a) $\operatorname{Re}_{\mathrm{e}}=0.5\left(\operatorname{Re}_{1}=1\right)$, (b) $\operatorname{Re}_{\mathrm{e}}=27.8$ ( $\left.\operatorname{Re}_{1}=60\right)$, and (c) $\operatorname{Re}_{\mathrm{e}}=55.7\left(\operatorname{Re}_{1}=120\right)$ [crosses; viscous: and solid bullets; pressure].

\section{Reynolds-number dependency}

The Reynolds-number dependency of drag, lift, and torque coefficients at moderate and high incidence angles are compared in Fig. 9. Although the drag coefficient behavior remains similar, the lift and torque coefficients of a 1:10 spheroid behave differently with $\mathrm{Re}$ at high incidence angles. At $\Phi \lesssim 80^{\circ}$ the lift and torque coefficients of all three spheroids decrease monotonically with increasing Reynolds numbers. At higher incidence angles, however, lift and torque coefficients of a 1:10 spheroid have a local minimum at $\operatorname{Re}_{\mathrm{e}} \approx$ 40. The two higher-aspect-ratio spheroids do not exhibit this behavior.

To illustrate the reason behind this different behavior at lower aspect ratios, in Fig. 10, we compare the contributions of viscous stress and pressure to the lift force and the pitching torque on a $1: 10$ spheroid at $\Phi=85^{\circ}$. The contribution from the pressure is dominant for both lift and pitching torques. Regardless of the incidence angle and particle Reynolds number, the viscous contribution to the pitching torque remains below $15 \%$. The pressure force is, therefore, the dominant contributor to the pitching torque. Concerning the lift force, at low and moderate incidence angles $(\Phi<80)$, the pressure and viscous stress contributions have counteracting effects. Regardless of the incidence angle, the relative contribution of the pressure force is dominant. At $\mathrm{Re}_{\mathrm{e}}=0.5$, for example, $\mid F_{\mathrm{L}}$, pressure $|\approx 1.7| F_{\mathrm{L} \text {,viscous }} \mid$ for all $\Phi$ 's. Furthermore, its contribution increases with Reynolds number up to $\operatorname{Re}_{\mathrm{e}} \approx 40$. At $\mathrm{Re}_{\mathrm{e}} \approx 40$ the viscous contribution to the lift force changes sign at $\Phi=85^{\circ}$. When the Reynolds number is increased further to $\operatorname{Re}_{\mathrm{e}}=55.7$ at $\Phi=85^{\circ}$ the relative contribution of the pressure to the lift force is about $80 \%$. These observations imply that the different behaviors of lift and torque coefficients for the 1:10 spheroid mostly stems from the pressure distribution at the surface of the particle.

Figure 11 compares the pressure field and the streamlines constructed based on the $x$ and $y$ components of velocity field at $z / d=0$ for four different combinations of incidence angle and Reynolds number. By comparing Figs. 11(c) and 11(d) corresponding to $\left(\operatorname{Re}_{\mathrm{e}}, \Phi\right)=\left(37.1,85^{\circ}\right)$ and $\left(\operatorname{Re}_{\mathrm{e}}, \Phi\right)=$ $\left(55.7,85^{\circ}\right)$, respectively, one can observe that for these two Reynolds numbers the upstream pressure profiles at the surface of the particle are very similar and almost symmetric in $y$, whereas for the downstream high-pressure regions this is not the case. At $\operatorname{Re}_{\mathrm{e}}=37.1$ the recirculation region is smaller and is located closer to the particle center. Due to the larger asymmetry of the flow field at $\mathrm{Re}_{\mathrm{e}}=55.7$, the recirculation area is shifted in $y$ direction farther away from the particle center. Furthermore, the velocity in the wake just behind the particle is dominantly oriented in positive $y$ direction, leading to a positive viscous force in this direction. These observations explain the existence of local minima for pitching torque and lift coefficients at $\Phi=85^{\circ}$.

The different behaviors of drag, lift, and pitching torque coefficients of the 1:10 spheroid observed confirm the necessity to derive shape-specific correlations for low-aspect-ratio spheroids. The dependencies for the 1:10 spheroid cannot be easily modeled by the symmetric $\sin ^{2}$

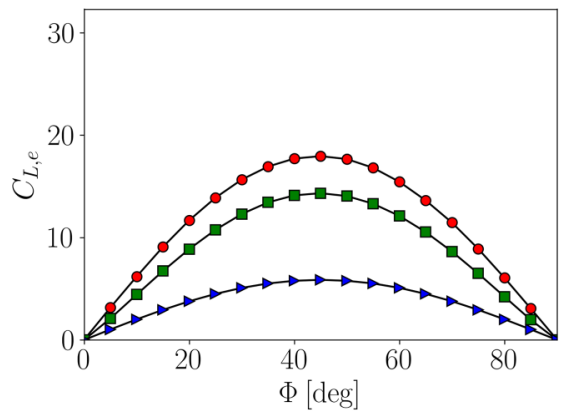

(a)

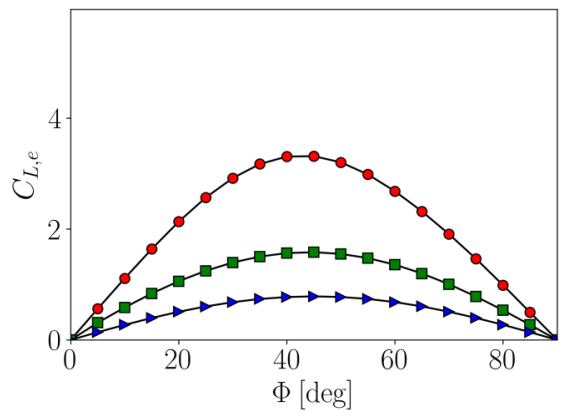

(b)

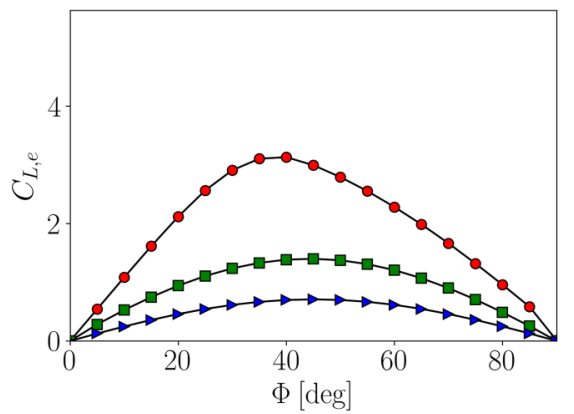

(c)

FIG. 7. Lift coefficient as a function of incidence angle at (a) $\operatorname{Re}_{\mathrm{e}}=0.5$, (b) $\operatorname{Re}_{\mathrm{e}}=27.8$, and (c) $\operatorname{Re}_{\mathrm{e}}=55.7$ [red solid bullet; $w_{1}=1 / 10$ : green solid square; $w_{2}=1 / 5$ : (solid small right triangle); $w_{3}=2 / 5$ ]. 


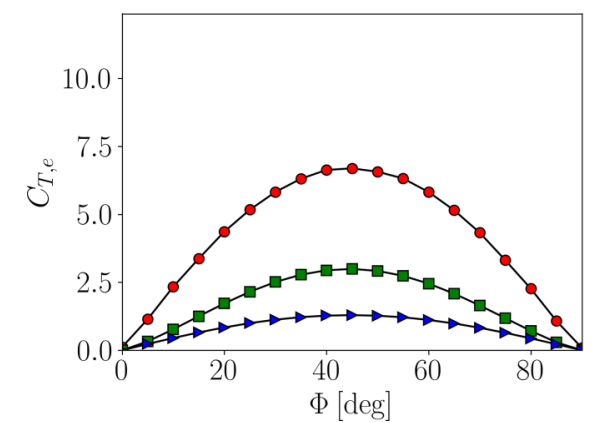

(a)

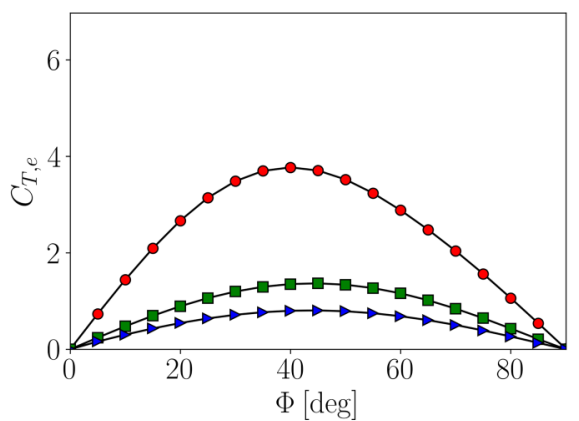

(b)

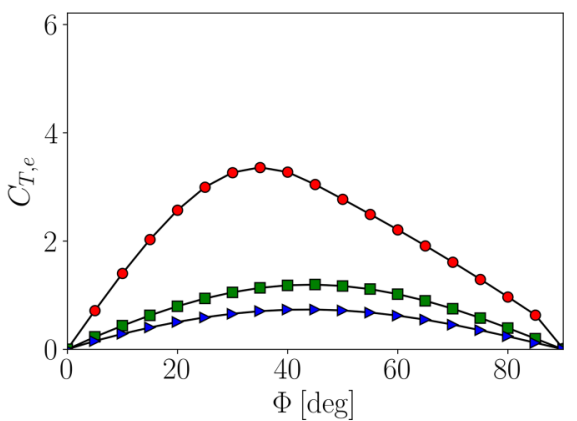

(c)

FIG. 8. Pitching torque coefficient as a function of incidence angle at (a) $\operatorname{Re}_{\mathrm{e}}=0.5$, (b) $\operatorname{Re}_{\mathrm{e}}=27.8$, and (c) $\operatorname{Re}_{\mathrm{e}}=55.7$ [red solid bullet; $w_{1}=1 / 10$ : green solid square; $w_{2}=1 / 5$ : (solid small right triangle); $\left.w_{3}=2 / 5\right]$.

correlations often used in literature at higher aspect ratios. To achieve an accurate analytical correlation, the fitting procedure can be very cumbersome as it requires introducing several additional coefficients to a correlation (as, for example, Ref. [28]). A neural network-based prediction, on the other hand, does not require such a priori information as it intrinsically recognizes and learns the different behaviors.

\section{Correlation procedure}

Upon completion of all simulations an input-output correlation has to be found in the form

$$
\boldsymbol{y}=\Theta(\boldsymbol{x}, \boldsymbol{\theta}),
$$

where $\boldsymbol{x}$ and $\boldsymbol{y}$ are the arrays of input and output, respectively, and $\boldsymbol{\theta}$ is the array of unknown parameters (weights and biases). Conventional regression (curve fitting) methods make an assumption on the structure of the function $\boldsymbol{\Theta}$ and find the unknown parameters through a minimization procedure. Finding a proper function (or a combination of functions) which captures all relevant features of the actual input-output relation can be cumbersome. Instead, we use deep learning to find an appropriate functional relationship and its corresponding weights and biases.

The basic idea of deep learning is to replicate an unknown complex function from known input and the corresponding output. A complex function is approximated by a combination of simpler functions which results in a so-called DNN. Through a deep learning process, the optimal parameters are found by minimizing (or maximizing) a target function. An example of such a target function is the difference between the output predicted by the neural network and the target
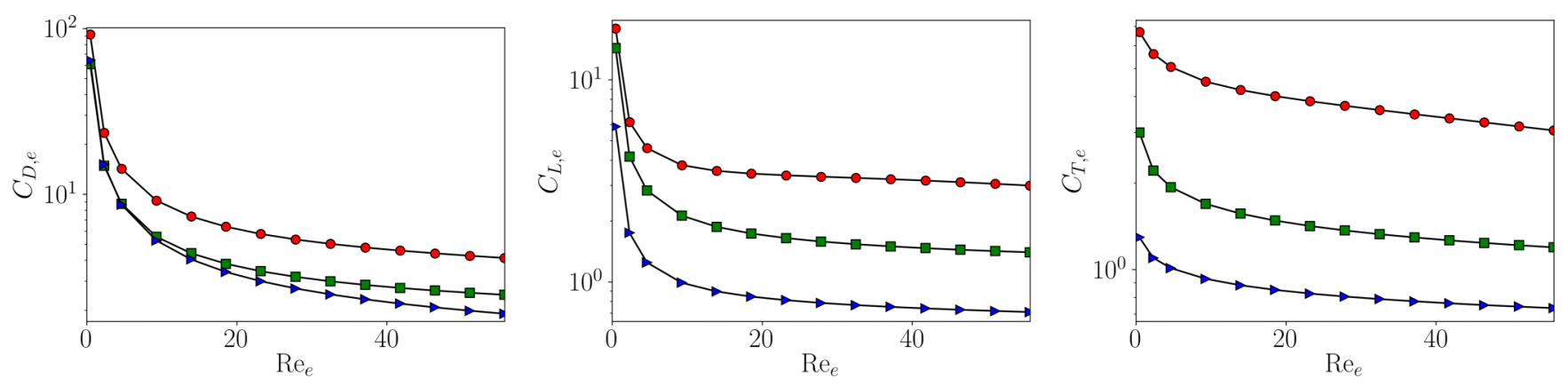

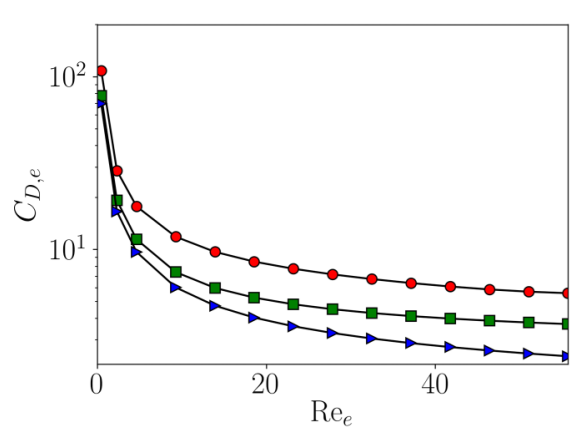

(a)

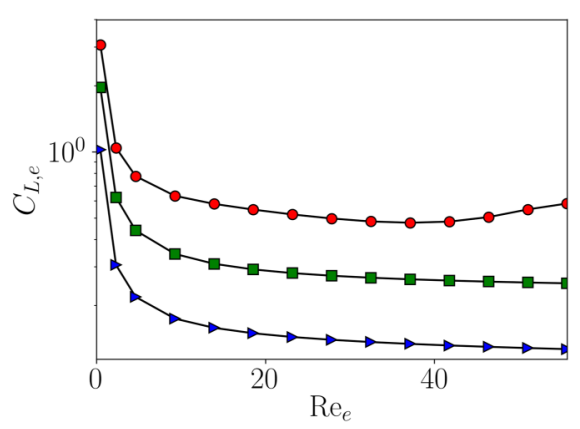

(b)

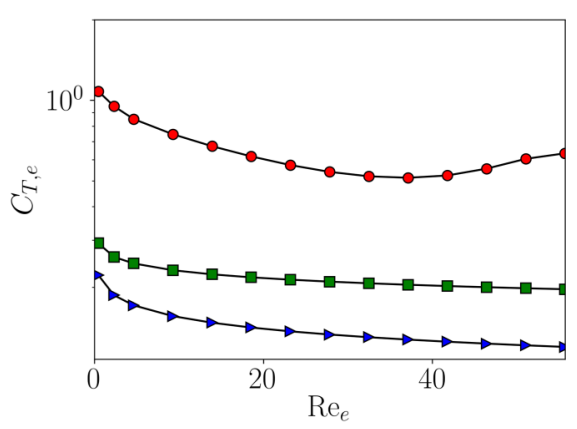

(c)

FIG. 9. Coefficients of (a) drag, (b) lift, and (c) pitching torque as a functions of Reynolds number at $\phi=45^{\circ}$ (top) and $\phi=85^{\circ}$ (bottom) incidence angles [red solid bullet; $w_{1}=1 / 10$ : green solid square; $w_{2}=1 / 5$ : (solid small right triangle); $w_{3}=2 / 5$ ]. 

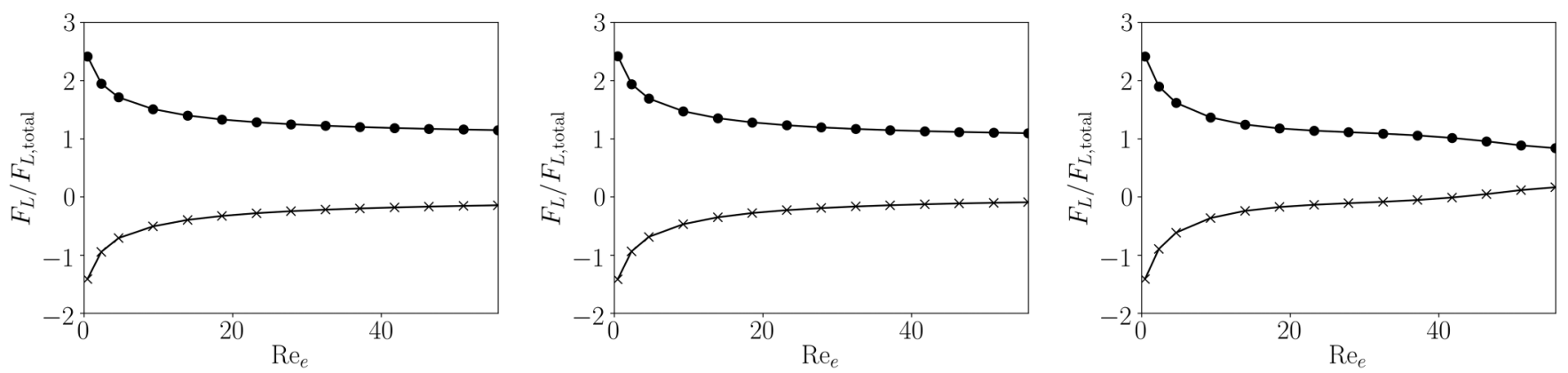

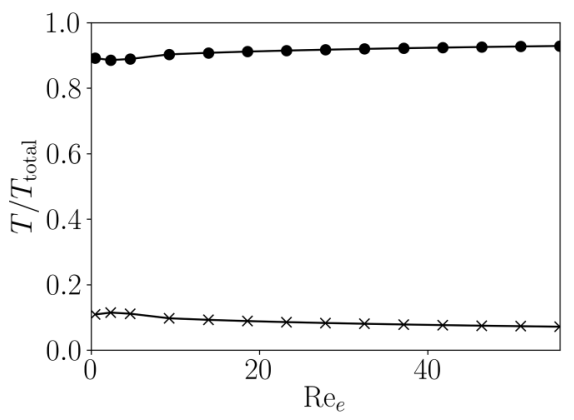

(a)

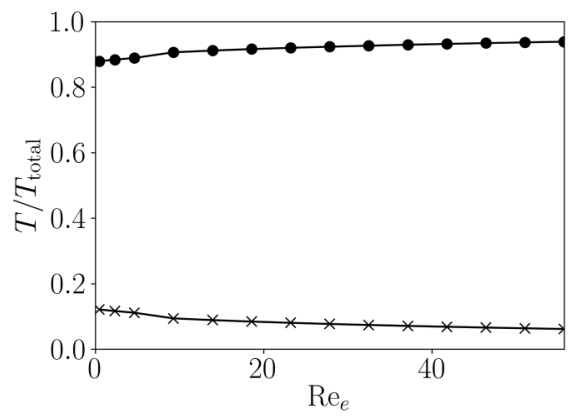

(b)

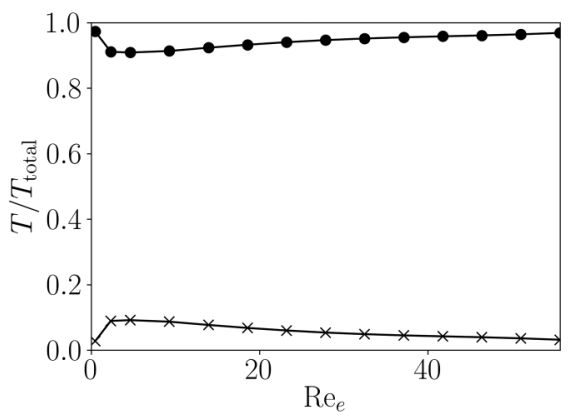

(c)

FIG. 10. Viscous and pressure contributions to the lift force (top) and pitching torque (bottom) on a 1:10 spheroid at (a) $\Phi=5^{\circ}$, (b) $\Phi=$ $30^{\circ}$, and (c) $\Phi=85^{\circ}$ crosses; viscous: and solid bullets; pressure].

value. This is the basic principle of one of the most applied neural network optimization approaches, the gradient descent method.

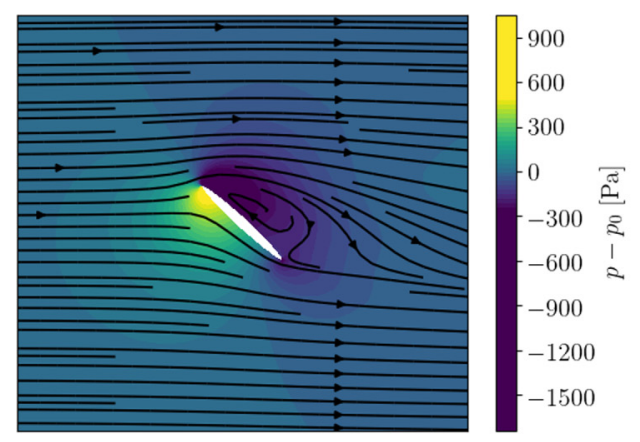

(a)

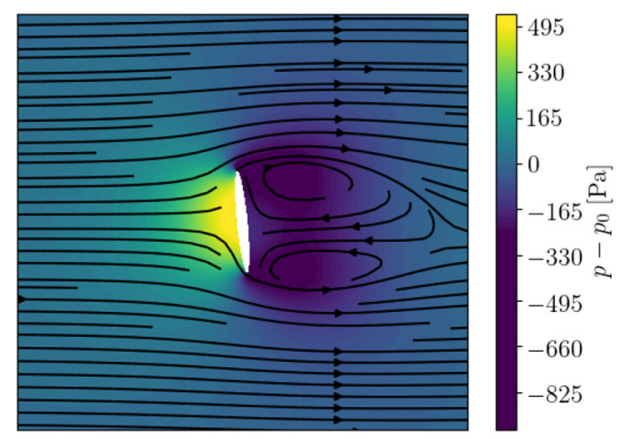

(c)
A DNN consists of multiple layers with several neurons. Every neuron is associated with one piece of information, its activation value. In each layer of a DNN, an input vector

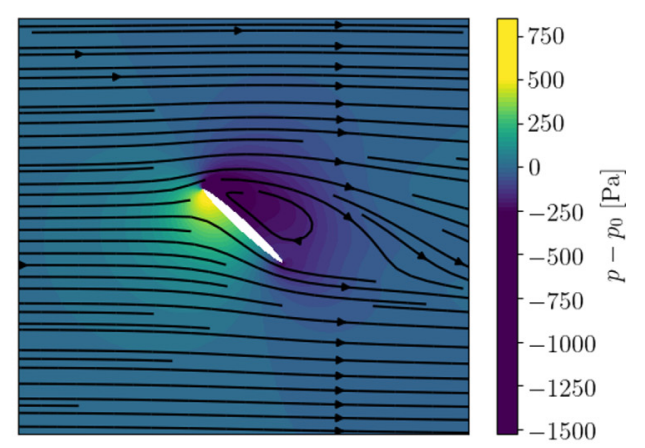

(b)

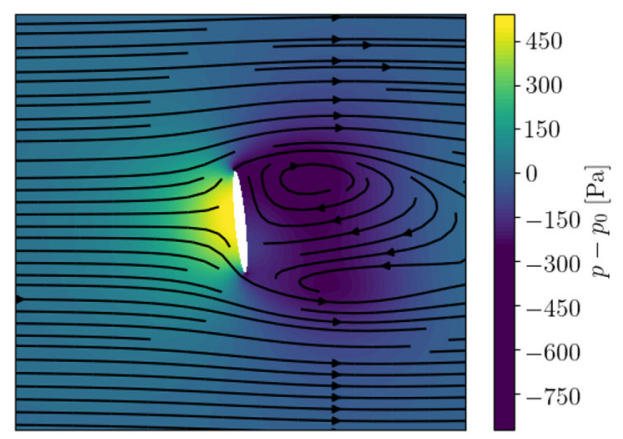

(d)

FIG. 11. Pressure field and streamline pattern in the center plane $z=0$ for the flow around a 1:10 spheroid at $(\mathrm{a})\left(\operatorname{Re}_{\mathrm{e}}, \Phi\right)=\left(37.1,45^{\circ}\right)$, (b) $\left(\operatorname{Re}_{\mathrm{e}}, \Phi\right)=\left(55.7,45^{\circ}\right),(\mathrm{c})\left(\operatorname{Re}_{\mathrm{e}}, \Phi\right)=\left(37.1,85^{\circ}\right)$, and (d) $\left(\operatorname{Re}_{\mathrm{e}}, \Phi\right)=\left(55.7,85^{\circ}\right)$. 


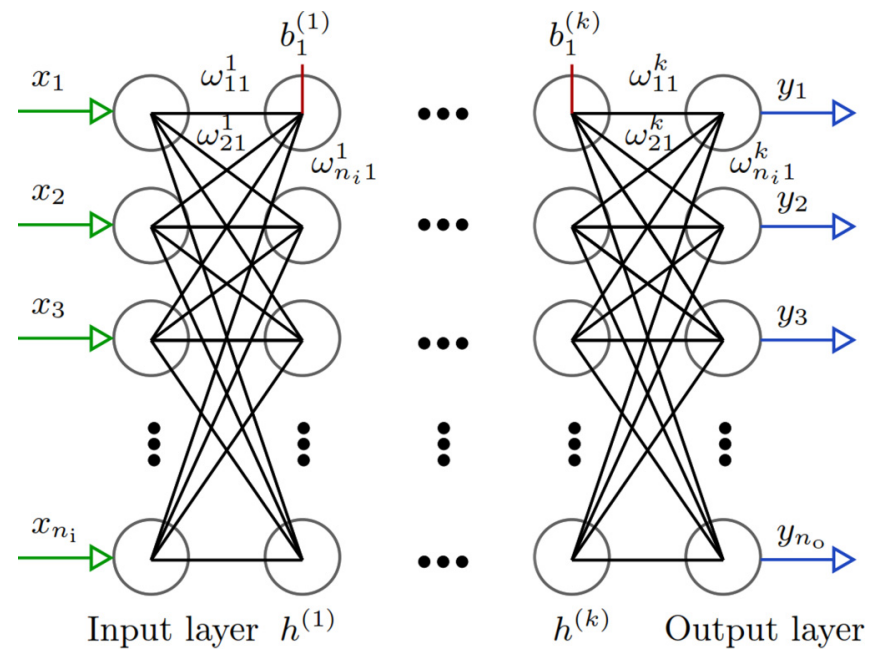

FIG. 12. Graphical representation of the architecture of a typical feedforward neural network with $n_{\mathrm{i}}$ inputs, $n_{\mathrm{o}}$ outputs, and $k$ hidden layers each consisting of $n_{k}$ neurons.

undergoes an elementwise nonlinear transformation which consists of a linear transformation through weights and biases followed by a nonlinear activation through the so-called "activation function." The activation values of the neurons in the first layer (input layer) are defined by the input data set. In the output layer, activation values are based on network connections. Neurons belonging to interior layers, or the so-called hidden layers, are connected to the neighboring layers via the weights and biases corresponding to each neuron. One famous representative of deep learning is the feedforward DNN, also known as a multilayer perceptron. In a feedforward DNN, the information propagates from the input layer through the hidden layers to the output layer. The basic architecture of a feedforward DNN is depicted in Fig. 12. For a network with $l$ hidden layers the activation values of the $k$ th hidden layer read

$$
h_{j}^{(1)}=f^{(1)}\left(\sum_{i=1}^{n_{k}} \omega_{i j}^{(1)} x_{i}+b_{j}^{(1)}\right),
$$

for $k=1$ and

$$
h_{j}^{(k)}=f^{(k)}\left(\sum_{i=1}^{n_{k}} \omega_{i j}^{(k)} h_{i}^{(k-1)}+b_{j}^{(k)}\right)
$$

for $2 \leqslant k \leqslant l-1$, where $f^{(k)}(x)$ is the activation function and $\omega_{i j}^{(k)}$ and $b_{j}^{(k)}$ are the weights and biases of the $k$ th layer. $n_{k}$ is the number of neurons in the $k$ th layer.

The weights and biases are found through a training procedure. During the training process, the weights and biases are changed to achieve an arbitrarily minimal difference between training data and the network predictions and, thus, also a preferably accurate estimation of unknown weight and bias values. A loss function $j$ is defined to quantify the quality of the predictions. One possibility for a loss function is the mean squared error between the predictions and the actual output values. The most common ways to minimize this loss are based on the iterative gradient-based optimization algorithm gradient descent. A vector $\boldsymbol{\theta}$, which represents weights or biases, is updated as follows:

$$
\boldsymbol{\theta}_{\mathrm{t}+1}=\boldsymbol{\theta}_{\mathrm{t}}-\eta \nabla_{\theta} j\left(\boldsymbol{x}, \boldsymbol{y}_{d}, \boldsymbol{\theta}_{\mathrm{t}}\right)
$$

where $\nabla_{\theta} j$ denotes the gradient of the loss function with respect to $\boldsymbol{\theta}$. The gradient itself depends on the input values $\boldsymbol{x}$, the desired outputs $\boldsymbol{y}_{d}$, and the other parameters $\boldsymbol{\theta}_{\mathrm{t}}$.

The parameter $\eta$ controls the learning rate by changing the step sizes of the updates. Its value should be chosen such that the iterative procedure finds small local minima of the loss function. Too small learning rates yield too slow optimization and the risk of getting stuck in large local minima. Too large learning rates may cause oscillations around a minimum. To avoid this, optimizers are derived that dynamically determine the learning rate [49]. The so-called Adam optimizer has been shown to provide beneficial convergence properties. This algorithm converges faster, and to a lower minimum than competitors, such as AdaGrad or AdaFom [50]. The Adam optimizer varies the step size based on the history of the gradients and the squared gradients by means of an exponential moving average. A detailed description of the algorithm can be found in the original publication of Kingma and $\mathrm{Ba}$ [51].

Besides the optimizer some additional aspects of a DNN have to be considered. First, the weights and biases have to be initialized in a certain way. In the present neural network the weights are initialized as small random values and the biases as zero. A common problem in the training process is so-called overfitting, which occurs when the neural network predicts the training values precisely but loses its accuracy with a new dataset. To avoid this, regularizers are implemented. The initialization, the effect of overfitting and different ways of regularization are described in detail by Goodfellow et al. [41].

Additionally, the activation function, the number of hidden layers, and the number of neurons in every hidden layer are application-specific parameters (hyperparameters), which influence the performance of the network. Especially finding the appropriate activation function without testing it is almost impossible since its influence is not fully understood yet [41].

In order to validate the trained DNN, out of the total data acquired from 266 simulations, 30 randomly selected simulation data points are excluded from the training data and are reserved for the validation. This way, the neural network is validated with data it has not seen before. The random sampling of the data for validation is repeated to make sure that the network prediction performance is not dependent on the random choice of the training data sample.

The considered neural network in the present paper consists of three hidden layers with 300 neurons. That means there are approximately $4 \times 10^{5}$ floating point operations per prediction. This number can be reduced by decreasing the number of neurons. With more experience, this is probably possible without a significant loss of accuracy by careful variation of the hyperparameters. The neurons of the hidden layers are activated by the so-called rectified linear (ReLU) activation functions. The outputs of the ReLU function are zero for negative inputs and equal the input for positive inputs [41]. Input and output layers have a linear activation function. The mean absolute error between predicted outputs and simulation data is minimized by an Adam optimizer. A L2 parameter norm penalty is used for the regularization. The network is 


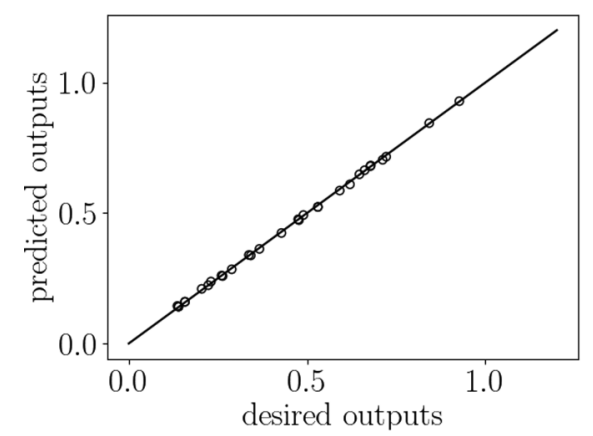

(a)

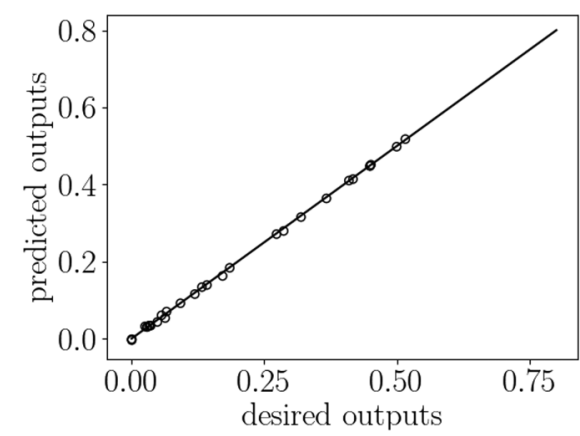

(b)

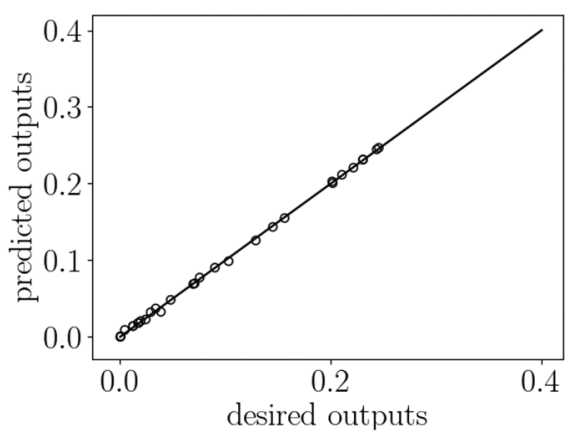

(c)

FIG. 13. Validation of the neural network prediction for (a) drag, (b) lift, and (c) pitching torque coefficients [(solid line); perfect network: (open bullet); present network].

trained 500 epochs with the 236 training data points in minibatches of size 32 . This means that an update is applied after 32 data points until every data point is used 500 times. The training of the DNN is performed by KERAS, an extension of the open-source machine learning library TENSORFLOW [52].

\section{DNN validation}

The performance of the network in predicting drag, lift, and pitching torque coefficients using the validation data set is illustrated in Fig. 13. The overall mean absolute error (mae) for the lift, drag, and the pitching torque coefficients is $L_{\mathrm{mae}}=0.0034$. The mean error for the coefficients of lift and pitching torque is below $3 \%$. The mean error for the drag coefficient is as low as $1.5 \%$. The smaller absolute values for the lift and torque coefficients compared to those of the drag coefficient yields larger relative errors for the lift and torque coefficients. Further optimization of the network may reduce these errors even more, but our results are comparable with Sanjeevi et al. who reported mean deviations of $1.66 \%$, $3.50 \%$, and $3.43 \%$ for their empirical correlations derived for drag, lift, and torque coefficient of a 2:5 spheroid, respectively.

\section{E. Testing the drag coefficient}

The weights and biases obtained through the training process can be found in the Supplemental Material [53]. These weights and biases are used to construct a DNN correlation according to (15). In this section, we test the interpolation and extrapolation performances of the DNN correlation for drag coefficient at $\Phi=90^{\circ}$ by comparing the DNN predictions with the correlation of Clift et al. [1] for thin disks. In Fig. 14 we compare our results with correlation (10) within the Reynolds-number range of $\operatorname{Re} \in[0,133]$. Although the lowest Reynolds number used for the training is $\mathrm{Re}=1$, it can be seen that the DNN model can very well capture the Stokes regime $(\operatorname{Re} \ll 1)$ behavior of the drag coefficient described by (11). We attribute this to the fact that the contribution of inertial effects to the drag coefficient at $\operatorname{Re} \approx 1$ is small.

\section{F. Application to a settling problem}

In this section, we employ the DNN-based drag correlation in a point-particle simulation of the buoyancy-driven motion of a 1:10 disk in a quiescent paramagnetic liquid in a nonuniform magnetic field. The buoyancy-driven motion of disks and ellipsoidal particles in viscous liquids has extensively been explored [54-56]. It is well known that the buoyancy-driven motion of disk-shaped particles can be fully described by three parameters, namely, the mass density ratio $\rho_{\mathrm{p}} / \rho_{\mathrm{f}}$, the aspect ratio $w$, and the Galileo number $(\mathrm{Ga})$ $\mathrm{Ga}=\sqrt{\left|1-\rho_{\mathrm{p}} / \rho_{\mathrm{f}}\right| g d_{\mathrm{p}}^{3}} / \nu_{\mathrm{f}}$, where $\rho_{\mathrm{f}}$ and $\rho_{\mathrm{p}}$ denote the mass densities of the fluid and particle, respectively, $v_{\mathrm{f}}$ is the fluid kinematic viscosity, and $g$ is the magnitude of gravitational acceleration $[1,54,56]$. Depending on the combination of these three nondimensional parameters, four main regimes for the settling motion of a disk are identified. At low Ga numbers where viscous effects are large enough, a disk falls broadside along a straight vertical path. As inertial effects increase, a disk can display a fluttering motion, and as the inertia further increases, tumbling and even full rotations may occur.

By incorporating a magnetically responsive liquid and a vertical magnetic field gradient, a nonlinear pressure is generated inside the liquid [57]. Once released in the liquid at $\Phi=90^{\circ}$, a disk with an adequately chosen mass density,

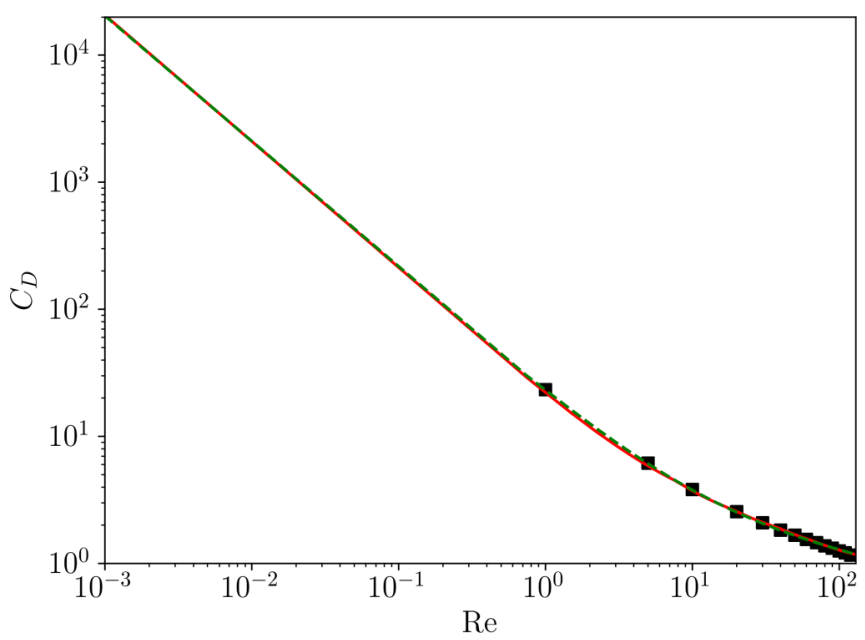

FIG. 14. Reynolds-number dependency of the steady drag coefficient of the 1:10 spheroid at $\Phi=90^{\circ}$ predicted by the DNN model is compared to correlation (10) (solid red line). The green dashed line represents the correlation of Clift et al. [1], and $\mathbf{\square}$ indicates the training data points. 
stably levitates at the height where the gravity force cancels the net buoyancy force acting on the particle. The existence of a stable equilibrium point in such a configuration makes the prediction of the time-dependent trajectory of a particle a good benchmark case for testing the DNN correlation within the Reynolds number range of $\operatorname{Re} \in[0,10]$.

The magnetically induced nonlinear pressure field inside the liquid leads to a position-dependent net buoyancy force on an immersed particle. The magnitude of this buoyancy force is dependent on the local gradient of the magnetic field $\nabla H$, and the magnetization of the liquid $M$ [58]. In the experiment, the gradient of the generated magnetic field is vertical $\frac{\partial H}{\partial x}=$ $\frac{\partial H}{\partial z}=0$ so that the combined gravitational and buoyancy force $(\mathrm{BG})$ is

$$
F_{\mathrm{BG}}=\left(\rho_{\mathrm{f}, \mathrm{a}}-\rho_{\mathrm{p}}\right) V_{i} g .
$$

Here, $\rho_{\mathrm{f}, \mathrm{a}}$ is the so-called "apparent mass density" of the magnetic liquid fluid defined as

$$
\rho_{\mathrm{f}, \mathrm{a}}(y)=\rho_{\mathrm{f}}-\frac{\mu_{0}}{g y} \int_{0}^{y} M \frac{d H}{d y} d y,
$$

where $\mu_{0}$ is the permeability of vacuum and $g$ is the magnitude of gravitational acceleration [57]. For a paramagnetic liquid at relatively low magnetic field strengths, the magnitude of the magnetization is a linear function of the magnitude of the magnetic field strength $M=\chi H$, where $\chi$ denotes the magnetic susceptibility of the liquid [59]. For small particles, the integral in (18) can be approximated by

$$
\rho_{\mathrm{f}, \mathrm{a}}(y) \approx \rho_{\mathrm{f}}-\left(\frac{\mu_{0}}{g}\right) M \frac{d H}{d y} .
$$

Once a particle with mass density $\rho_{\mathrm{p}}$ is released in the fluid at initial height $y_{0}$, it travels to a height at which $\rho_{\mathrm{f}, \mathrm{a}}(y)=\rho_{\mathrm{p}}$.

The incorporated magnet system generates a magnetic field that has a magnitude decaying exponentially with the vertical distance from the magnet surface $y$,

$$
H(y)=H_{0} e^{-\pi y / p},
$$

with $H_{0}=422 \mathrm{kA} / \mathrm{m}$ and pole size $p=0.118 \mathrm{~m}$. The magnetic field strength and the apparent mass density of the liquid according to (19) are plotted as functions of $y$ in Fig. 15. The vertical position of the particle is recorded by a three dimensional particle tracking velocimetry technique. Measurements are performed in a $15 \times 15 \times 15 \mathrm{~cm}^{3}$ cubic container filled with a stable aqueous solution of $\mathrm{MnCl}_{2}$. The container is located on a magnet that generates the desired magnetic field in the form of (20). Two cameras record the particle trajectories through two perpendicular sidewalls of the tank. The particle considered for the experiments is a disk made of unplasticized polytetrafluorethylene $\rho_{\mathrm{p}}=1434 \mathrm{~kg} / \mathrm{m}^{3}$. A schematic of the experimental setup is shown in Fig. 16.

We employ a one-way coupled point-particle model where the effect of the particle motion on the fluid is assumed to be negligible. The motion of a the particle is described by solving the translational equation of motion of the particle. An angular momentum equation is not solved for the following reason: In the considered magnetofluidic system an apparent Galileo number can be defined as $\mathrm{Ga}_{\mathrm{a}}=\sqrt{\left|1-\rho_{\mathrm{p}} / \rho_{\mathrm{f}, \mathrm{a}}\right| g d_{\mathrm{p}}^{3}} / \nu_{\mathrm{f}}$. In the present configuration with $\rho_{\mathrm{p}}=1.434 \times 10^{3} \mathrm{~kg} / \mathrm{m}^{3}$, the

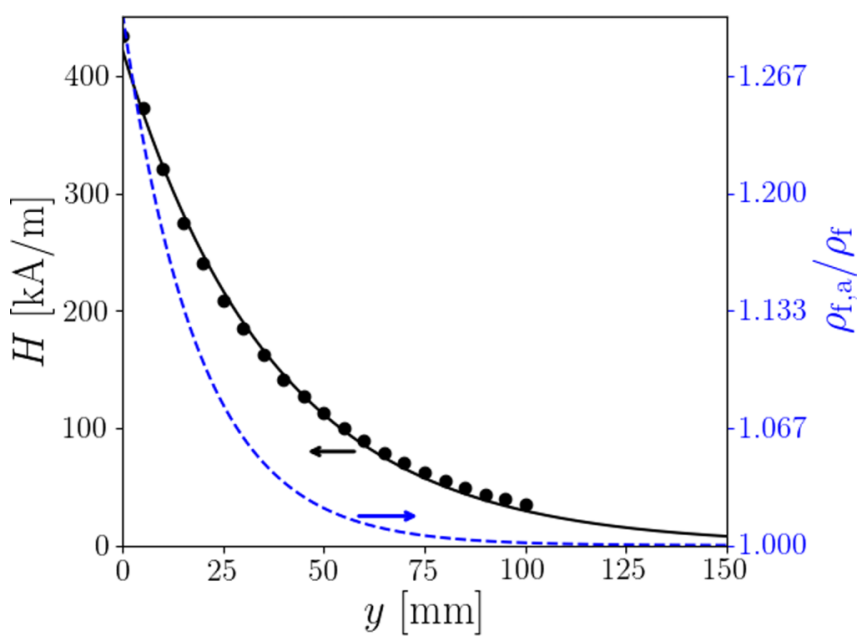

FIG. 15. Magnetic field strength and effective mass density on a vertical line passing through the center of the tank. A particle with $\rho_{\mathrm{p}} / \rho_{\mathrm{f}}=1.02$ stably levitates at $y=48.7 \mathrm{~mm}$ [(solid black line); exponential fit; (solid bullet); measured)].

maximum apparent Galileo number of a particle remains below 70. The motion of a $1: 10$ disk with $\rho_{\mathrm{p}} / \rho_{\mathrm{f}} \approx 1$ and $\mathrm{Ga}_{\mathrm{a}} \leqslant 70$ falling at $\Phi=90^{\circ}$ is steady and follows a straight vertical path, and the particle does not undergo any rotational motion. Hence, the governing equations of the particle motion reduces to a scalar system of the form

$$
\begin{aligned}
& \frac{d y}{d t}=v, \\
& \frac{d v}{d t}=\frac{1}{m_{\mathrm{p}}} \sum F,
\end{aligned}
$$

where $\sum F$ is the sum of all forces stemming from different fluid-particle hydrodynamic interactions and the gravity force. The relevant forces in the motion of almost neutrally buoyant particles in viscous liquids are the steady drag force $F_{\mathrm{D}}$, the combined buoyancy and gravity force $F_{\mathrm{BG}}$, the history force $F_{\mathrm{H}}$, and the added mass force $F_{\mathrm{A}}$ so that

$$
\sum F=F_{\mathrm{D}}+F_{\mathrm{BG}}+F_{\mathrm{H}}+F_{\mathrm{A}} \text {. }
$$

The steady drag force is given by

$$
F_{\mathrm{D}}=\frac{1}{2} C_{\mathrm{D}} \rho_{\mathrm{p}} v^{2} A_{\mathrm{p}},
$$

where $C_{\mathrm{D}}$ is the steady drag coefficient and $A_{\mathrm{p}}=\pi d^{2} / 4$ is the cross sectional area of the particle with equatorial diameter $d$.

For history force and added mass force, we neglect the finite Reynolds number effects and follow the expressions derived by Lai and Mockros [60] for a spheroid moving parallel to its symmetry axis. The history force reads

$$
F_{\mathrm{H}}=-6 \Delta_{\mathrm{H}}\left(\pi \rho_{\mathrm{f}} \mu\right)^{1 / 2} a^{2} \int_{-\infty}^{t} \frac{\frac{d v}{d \tau}}{(t-\tau)^{1 / 2}} d \tau,
$$

where the history force coefficient $\Delta_{\mathrm{H}}$ for a particle with aspect ratio $w$ is

$$
\Delta_{\mathrm{H}}=\left[\frac{4\left(1-w^{2}\right)}{3\left\{\left[\left(1-2 w^{2}\right) \arccos \left(w / \sqrt{1-w^{2}}\right)\right]+w\right\}}\right]^{2} .
$$




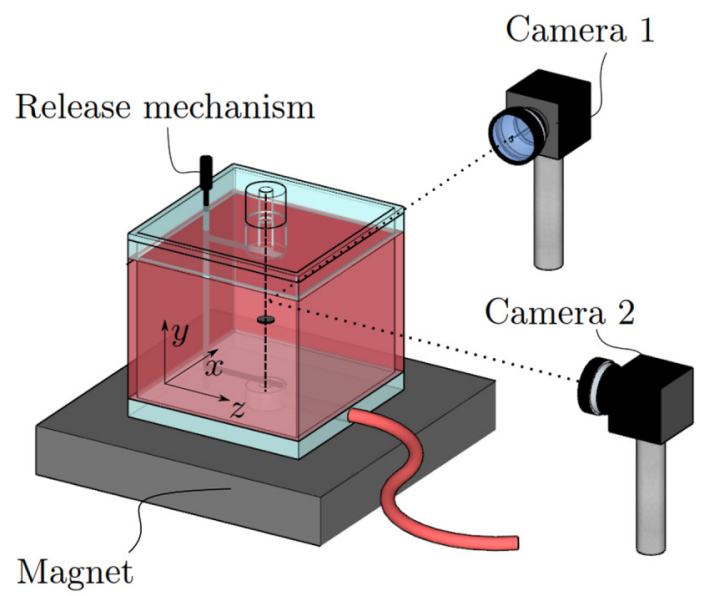

(a)

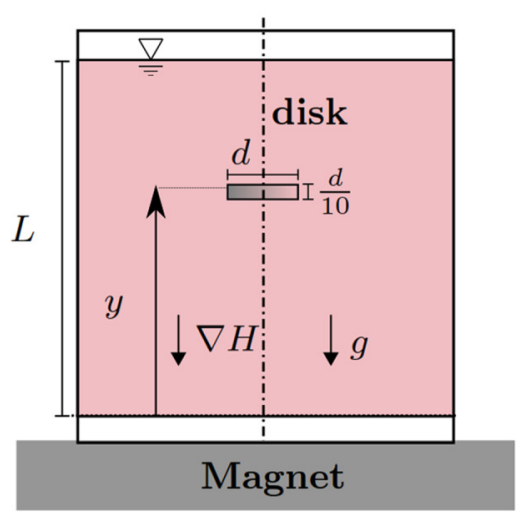

(b)

FIG. 16. (a) A sketch of the experimental setup. A tank filled with manganese(II) chloride solution is placed on top of a magnet. The particle is released from the top by a rotating release mechanism. Two cameras record the motion of the released particle. (b) Schematic side view.

The added mass force is given by

$$
F_{\mathrm{AM}}=-\frac{1}{2} \Delta_{\mathrm{A}} \rho_{\mathrm{f}} V \frac{d v}{d t},
$$

where $\Delta_{\mathrm{A}}$ is the added mass coefficient given by

$$
\Delta_{\mathrm{A}}=\frac{2\left[w \cos ^{-1} w-\sqrt{1-w^{2}}\right]}{w^{2} \sqrt{1-w^{2}}-w \cos ^{-1} w} .
$$

For a spheroid with $w=0.1$, Eqs. (26) and (27) yield, respectively, $\Delta_{\mathrm{A}}=12.4$ and $\Delta_{\mathrm{H}}=0.7$. It is notable that the errors introduced by neglecting the effects of finite Reynolds numbers on the history and and added mass forces are expected to be small since the particle is initially at rest, and its acceleration rate is relatively low in the experiments.

To obtain the temporal evolution of the vertical particle position, system (21) is discretized using an explicit Euler scheme. For numerical integration of the Basset history term, we use the method of Van Hinsberg et al. [61] where the history kernel is split into a "window" kernel and a "tail" kernel. The window kernel is approximated by a trapezoidal rule, and the tail kernel is approximated by a sum of exponential functions. We test the NN-based drag model by comparing the particle trajectory based on the DNN-based drag model $C_{\mathrm{D}}\left(\operatorname{Re}, \Phi=90^{\circ}\right)$ with the experimentally obtained trajectory.

TABLE II. Physical parameters of the magnetic levitation setup.

\begin{tabular}{lc}
\hline \hline$\rho_{\mathrm{p}}\left(\mathrm{kg} / \mathrm{m}^{3}\right)$ & $1.434 \times 10^{3}$ \\
$d(\mathrm{~mm})$ & 5 \\
$b(\mathrm{~mm})$ & 0.5 \\
$\rho_{\mathrm{f}}\left(\mathrm{kg} / \mathrm{m}^{3}\right)$ & $1.403 \times 10^{3}$ \\
$\mu_{\mathrm{f}}(\mathrm{kg} \mathrm{m} / \mathrm{s})$ & $5.54 \times 10^{-3}$ \\
$\chi_{\mathrm{f}}$ & $7 \times 10^{-4}$ \\
$L(\mathrm{~m})$ & 0.15 \\
$p(\mathrm{~m})$ & 0.1181 \\
$H_{0}(\mathrm{kA} / \mathrm{m})$ & 422 \\
\hline \hline
\end{tabular}

Table II summarizes the parameters of the considered configuration.

A particle with mass density $\rho_{\mathrm{p}}=1.02 \rho_{\mathrm{f}}$ stably levitates at $y=48.7 \mathrm{~mm}$. Figure 17 compares the numerically and experimentally obtained trajectories of the particle released at $y_{0}=104.2 \mathrm{~mm}$. The experimental trajectory is obtained by averaging the results of three independent experiments. The error bars correspond to the standard deviation of these three measurements. The maximum particle Reynolds number during the levitation motion is $\mathrm{Re}=7.6$. Very good agreement is observed between the experimental trajectory and the one obtained with the point-particle simulation based on the DNN-based drag correlation. This observation shows the promising performance of the DDN model in predicting the drag coefficient of a 1:10 disk.

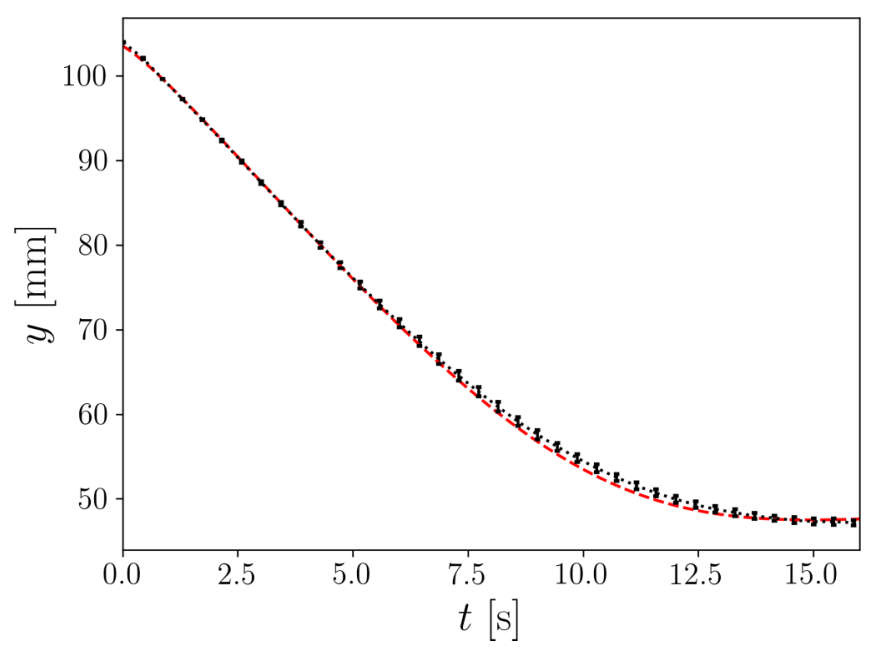

FIG. 17. Vertical position of a 1:10 disk in a magnetized paramagnetic liquid as a function time. The red dashed line corresponds to the numerical solution of (21) with the DNN-based drag coefficient. To enable the comparison, the numerical results are slightly shifted in time. The experimentally obtained trajectory is indicated by the dotted line. 
TABLE III. Coefficient of drag $C_{\mathrm{D}, \mathrm{e}}$. Leftmost column: incidence angle, top row: Reynolds number $\mathrm{Re}_{\mathrm{e}}$, and center: values of $C_{\mathrm{D}, \mathrm{e}}$.

\begin{tabular}{|c|c|c|c|c|c|c|c|c|c|c|c|c|c|c|}
\hline Drag & 0.46 & 2.32 & 4.64 & 9.28 & 13.92 & 18.57 & 23.21 & 27.85 & 32.49 & 37.13 & 41.77 & 46.42 & 51.06 & 55.7 \\
\hline 0.000 & 74.660 & 18.130 & 10.460 & 6.250 & 4.710 & 3.880 & 3.350 & 2.980 & 2.700 & 2.480 & 2.300 & 2.160 & 2.040 & 1.930 \\
\hline 5.000 & 74.920 & 18.210 & 10.510 & 6.300 & 4.750 & 3.910 & 3.380 & 3.010 & 2.730 & 2.510 & 2.340 & 2.190 & 2.070 & 1.960 \\
\hline 10.000 & 75.730 & 18.470 & 10.690 & 6.430 & 4.870 & 4.030 & 3.490 & 3.110 & 2.830 & 2.610 & 2.430 & 2.290 & 2.170 & 2.060 \\
\hline 15.000 & 77.020 & 18.870 & 10.980 & 6.640 & 5.060 & 4.210 & 3.660 & 3.280 & 3.000 & 2.780 & 2.600 & 2.450 & 2.330 & 2.220 \\
\hline 20.000 & 78.760 & 19.420 & 11.360 & 6.940 & 5.330 & 4.460 & 3.910 & 3.520 & 3.240 & 3.010 & 2.830 & 2.690 & 2.560 & 2.460 \\
\hline 25.000 & 80.850 & 20.080 & 11.830 & 7.300 & 5.660 & 4.770 & 4.210 & 3.820 & 3.530 & 3.310 & 3.130 & 2.980 & 2.860 & 2.750 \\
\hline 30.000 & 83.340 & 20.860 & 12.370 & 7.720 & 6.030 & 5.130 & 4.560 & 4.170 & 3.880 & 3.650 & 3.470 & 3.320 & 3.200 & 3.090 \\
\hline 35.000 & 86.080 & 21.710 & 12.970 & 8.180 & 6.450 & 5.530 & 4.950 & 4.550 & 4.250 & 4.020 & 3.840 & 3.690 & 3.560 & 3.460 \\
\hline 40.000 & 88.920 & 22.600 & 13.600 & 8.670 & 6.890 & 5.950 & 5.360 & 4.950 & 4.640 & 4.410 & 4.220 & 4.060 & 3.920 & 3.810 \\
\hline 45.000 & 91.850 & 23.500 & 14.230 & 9.170 & 7.340 & 6.370 & 5.760 & 5.340 & 5.020 & 4.780 & 4.580 & 4.410 & 4.260 & 4.130 \\
\hline 50.000 & 94.840 & 24.420 & 14.870 & 9.660 & 7.780 & 6.780 & 6.150 & 5.710 & 5.380 & 5.120 & 4.900 & 4.720 & 4.570 & 4.430 \\
\hline 55.000 & 97.620 & 25.280 & 15.480 & 10.130 & 8.200 & 7.170 & 6.510 & 6.050 & 5.700 & 5.430 & 5.200 & 5.010 & 4.850 & 4.720 \\
\hline 60.000 & 100.240 & 26.080 & 16.040 & 10.560 & 8.580 & 7.520 & 6.840 & 6.350 & 5.990 & 5.700 & 5.470 & 5.270 & 5.110 & 4.970 \\
\hline 65.000 & 102.660 & 26.810 & 16.550 & 10.940 & 8.910 & 7.820 & 7.110 & 6.610 & 6.230 & 5.930 & 5.690 & 5.490 & 5.330 & 5.200 \\
\hline 70.000 & 104.780 & 27.450 & 16.990 & 11.270 & 9.200 & 8.070 & 7.350 & 6.830 & 6.430 & 6.120 & 5.870 & 5.670 & 5.510 & 5.380 \\
\hline 75.000 & 106.400 & 27.940 & 17.340 & 11.530 & 9.410 & 8.260 & 7.520 & 6.990 & 6.580 & 6.250 & 6.000 & 5.790 & 5.630 & 5.500 \\
\hline 80.000 & 107.630 & 28.310 & 17.590 & 11.720 & 9.580 & 8.410 & 7.650 & 7.100 & 6.680 & 6.340 & 6.070 & 5.860 & 5.700 & 5.580 \\
\hline 85.000 & 108.330 & 28.520 & 17.740 & 11.840 & 9.680 & 8.500 & 7.730 & 7.170 & 6.730 & 6.390 & 6.100 & 5.860 & 5.690 & 5.580 \\
\hline 90.000 & 108.530 & 28.590 & 17.790 & 11.870 & 9.700 & 8.520 & 7.750 & 7.190 & 6.750 & 6.390 & 6.100 & 5.840 & 5.620 & 5.420 \\
\hline
\end{tabular}

\section{CONCLUSIONS}

Predicting steady hydrodynamic interactions of nonspherical particles becomes more challenging as the particle nonsphericity increases. The complexity of the input-output relations, on one hand, and the increased number of input parameters, on the other hand, make the development of empirical force and torque models very difficult. In this paper, we showed how statistical learning can serve as a versatile and robust tool for predicting hydrodynamic interactions of nonspherical particles with a viscous liquid. A machine learning approach is proposed to create models for the Reynoldsnumber- and incidence-angle-dependent steady drag, lift, and pitching torque coefficient of a 1:10 oblate spheroid. A feedforward deep neural network is trained and validated using a data set generated by resolved simulations at 261 different combinations of particle Reynolds number and incidence angle.

The effect of aspect ratio on the dependency of drag, lift, and pitching torque on the particle Reynolds number and incidence angle is illustrated, and advantages of a DNN-based correlation method compared to conventional curve-fitting methods are pointed out. It is shown that although the effect of aspect ratio on the behavior of the drag force is small, the lift and torque coefficients at low aspect ratios behave

TABLE IV. Coefficient of lift $C_{\mathrm{L}, \mathrm{e}}$. Leftmost column: incidence angle, top row: Reynolds number $\mathrm{Re}_{\mathrm{e}}$, and center: values of $C_{\mathrm{L}, \mathrm{e}}$.

\begin{tabular}{lllllllllllllll}
\hline \hline Lift & 0.46 & 2.32 & 4.64 & 9.28 & 13.92 & 18.57 & 23.21 & 27.85 & 32.49 & 37.13 & 41.77 & 46.42 & 51.06 & 55.7 \\
\hline 0.000 & $1 \cdot 10^{-2}$ & 0.000 & 0.000 & 0.000 & 0.000 & 0.000 & 0.000 & 0.000 & 0.000 & 0.000 & 0.000 & 0.000 & 0.000 & 0.000 \\
5.000 & 3.160 & 1.100 & 0.810 & 0.660 & 0.610 & 0.580 & 0.570 & 0.560 & 0.560 & 0.550 & 0.550 & 0.550 & 0.540 & 0.540 \\
10.000 & 6.200 & 2.150 & 1.600 & 1.290 & 1.200 & 1.150 & 1.130 & 1.110 & 1.100 & 1.090 & 1.090 & 1.090 & 1.080 & 1.080 \\
15.000 & 9.090 & 3.150 & 2.330 & 1.890 & 1.760 & 1.690 & 1.660 & 1.640 & 1.630 & 1.620 & 1.620 & 1.610 & 1.610 & 1.610 \\
20.000 & 11.690 & 4.050 & 3.000 & 2.440 & 2.270 & 2.190 & 2.150 & 2.130 & 2.120 & 2.110 & 2.110 & 2.110 & 2.110 & 2.120 \\
25.000 & 13.890 & 4.800 & 3.560 & 2.910 & 2.720 & 2.630 & 2.590 & 2.570 & 2.560 & 2.550 & 2.550 & 2.560 & 2.560 & 2.560 \\
30.000 & 15.660 & 5.410 & 4.020 & 3.290 & 3.070 & 2.980 & 2.940 & 2.920 & 2.910 & 2.900 & 2.900 & 2.900 & 2.910 & 2.910 \\
35.000 & 16.930 & 5.840 & 4.340 & 3.560 & 3.340 & 3.240 & 3.200 & 3.170 & 3.160 & 3.140 & 3.140 & 3.130 & 3.120 & 3.100 \\
40.000 & 17.710 & 6.100 & 4.530 & 3.730 & 3.500 & 3.390 & 3.340 & 3.310 & 3.280 & 3.250 & 3.220 & 3.190 & 3.160 & 3.130 \\
45.000 & 17.950 & 6.170 & 4.590 & 3.780 & 3.540 & 3.430 & 3.360 & 3.310 & 3.270 & 3.220 & 3.170 & 3.110 & 3.050 & 2.990 \\
50.000 & 17.670 & 6.060 & 4.500 & 3.710 & 3.470 & 3.350 & 3.270 & 3.200 & 3.130 & 3.060 & 2.990 & 2.920 & 2.850 & 2.790 \\
55.000 & 16.810 & 5.750 & 4.280 & 3.520 & 3.290 & 3.160 & 3.070 & 2.980 & 2.900 & 2.820 & 2.740 & 2.670 & 2.610 & 2.550 \\
60.000 & 15.440 & 5.280 & 3.920 & 3.230 & 3.000 & 2.870 & 2.770 & 2.680 & 2.590 & 2.510 & 2.440 & 2.380 & 2.330 & 2.280 \\
65.000 & 13.630 & 4.650 & 3.460 & 2.840 & 2.630 & 2.510 & 2.410 & 2.320 & 2.240 & 2.170 & 2.110 & 2.060 & 2.020 & 1.980 \\
70.000 & 11.460 & 3.900 & 2.900 & 2.370 & 2.190 & 2.080 & 1.990 & 1.910 & 1.840 & 1.780 & 1.740 & 1.710 & 1.690 & 1.660 \\
75.000 & 8.900 & 3.030 & 2.250 & 1.840 & 1.690 & 1.600 & 1.530 & 1.460 & 1.410 & 1.380 & 1.350 & 1.340 & 1.330 & 1.320 \\
80.000 & 6.070 & 2.060 & 1.530 & 1.250 & 1.150 & 1.090 & 1.030 & 0.990 & 0.960 & 0.940 & 0.930 & 0.940 & 0.950 & 0.960 \\
85.000 & 3.070 & 1.040 & 0.780 & 0.630 & 0.580 & 0.550 & 0.520 & 0.500 & 0.480 & 0.480 & 0.480 & 0.500 & 0.550 & 0.580 \\
90.000 & 1.10 & 0.000 & 0.000 & $1.10^{-2}$ & 0.000 & 0.000 & 0.000 & 0.000 & 0.000 & 0.000 & 0.000 & 0.000 & 0.000 & 0.000 \\
\hline \hline
\end{tabular}


TABLE V. Coefficient of torque $C_{\mathrm{T}, \mathrm{e}}$. Leftmost column: incidence angle, top row: Reynolds number $\mathrm{Re}_{\mathrm{e}}$, and center: values of $C_{\mathrm{T}, \mathrm{e}}$.

\begin{tabular}{|c|c|c|c|c|c|c|c|c|c|c|c|c|c|c|}
\hline Torque & 0.46 & 2.32 & 4.64 & 9.28 & 13.92 & 18.57 & 23.21 & 27.85 & 32.49 & 37.13 & 41.77 & 46.42 & 51.06 & 55.7 \\
\hline 0.000 & $3 \cdot 10^{-2}$ & $1 \cdot 10^{-2}$ & 0.000 & 0.000 & 0.000 & 0.000 & 0.000 & 0.000 & 0.000 & 0.000 & 0.000 & 0.000 & 0.000 & 0.000 \\
\hline 5.000 & 1.140 & 0.980 & 0.890 & 0.810 & 0.780 & 0.760 & 0.740 & 0.740 & 0.730 & 0.720 & 0.720 & 0.720 & 0.720 & 0.710 \\
\hline 10.000 & 2.330 & 1.940 & 1.760 & 1.600 & 1.530 & 1.490 & 1.460 & 1.440 & 1.430 & 1.420 & 1.410 & 1.410 & 1.400 & 1.400 \\
\hline 15.000 & 3.370 & 2.830 & 2.570 & 2.330 & 2.220 & 2.160 & 2.120 & 2.090 & 2.070 & 2.060 & 2.050 & 2.040 & 2.030 & 2.030 \\
\hline 20.000 & 4.360 & 3.640 & 3.300 & 2.990 & 2.850 & 2.760 & 2.710 & 2.670 & 2.640 & 2.620 & 2.600 & 2.590 & 2.580 & 2.570 \\
\hline 25.000 & 5.170 & 4.330 & 3.920 & 3.550 & 3.370 & 3.270 & 3.190 & 3.140 & 3.100 & 3.070 & 3.050 & 3.030 & 3.010 & 2.990 \\
\hline 30.000 & 5.820 & 4.890 & 4.420 & 3.980 & 3.770 & 3.640 & 3.550 & 3.490 & 3.430 & 3.390 & 3.350 & 3.320 & 3.290 & 3.270 \\
\hline 35.000 & 6.310 & 5.290 & 4.780 & 4.290 & 4.050 & 3.900 & 3.790 & 3.700 & 3.630 & 3.560 & 3.510 & 3.460 & 3.410 & 3.360 \\
\hline 40.000 & 6.630 & 5.550 & 5.000 & 4.460 & 4.200 & 4.020 & 3.880 & 3.770 & 3.680 & 3.590 & 3.500 & 3.420 & 3.350 & 3.270 \\
\hline 45.000 & 6.690 & 5.620 & 5.060 & 4.500 & 4.210 & 4.000 & 3.840 & 3.710 & 3.580 & 3.460 & 3.350 & 3.250 & 3.140 & 3.040 \\
\hline 50.000 & 6.570 & 5.520 & 4.970 & 4.400 & 4.090 & 3.870 & 3.680 & 3.520 & 3.370 & 3.230 & 3.100 & 2.980 & 2.870 & 2.770 \\
\hline 55.000 & 6.320 & 5.280 & 4.730 & 4.160 & 3.850 & 3.610 & 3.410 & 3.240 & 3.070 & 2.930 & 2.800 & 2.680 & 2.580 & 2.490 \\
\hline 60.000 & 5.820 & 4.860 & 4.340 & 3.810 & 3.500 & 3.260 & 3.060 & 2.880 & 2.720 & 2.590 & 2.470 & 2.370 & 2.280 & 2.210 \\
\hline 65.000 & 5.150 & 4.290 & 3.830 & 3.350 & 3.060 & 2.830 & 2.640 & 2.480 & 2.340 & 2.220 & 2.130 & 2.050 & 1.980 & 1.910 \\
\hline 70.000 & 4.320 & 3.600 & 3.210 & 2.790 & 2.540 & 2.340 & 2.180 & 2.040 & 1.930 & 1.840 & 1.770 & 1.710 & 1.660 & 1.610 \\
\hline 75.000 & 3.310 & 2.790 & 2.490 & 2.160 & 1.960 & 1.800 & 1.670 & 1.560 & 1.490 & 1.430 & 1.390 & 1.360 & 1.330 & 1.290 \\
\hline 80.000 & 2.270 & 1.910 & 1.700 & 1.470 & 1.330 & 1.220 & 1.130 & 1.060 & 1.010 & 0.990 & 0.980 & 0.980 & 0.980 & 0.970 \\
\hline 85.000 & 1.080 & 0.950 & 0.850 & 0.750 & 0.670 & 0.620 & 0.570 & 0.540 & 0.520 & 0.510 & 0.520 & 0.550 & 0.600 & 0.630 \\
\hline 90.000 & $8 \cdot 10^{-2}$ & $2 \cdot 10^{-2}$ & $1 \cdot 10^{-2}$ & $2 \cdot 10^{-2}$ & $2 \cdot 10^{-2}$ & $1 \cdot 10^{-2}$ & $1 \cdot 10^{-2}$ & $1 \cdot 10^{-2}$ & $1 \cdot 10^{-2}$ & $1 \cdot 10^{-2}$ & $1 \cdot 10^{-2}$ & $1 \cdot 10^{-2}$ & $1 \cdot 10^{-2}$ & $1 \cdot 10^{-2}$ \\
\hline
\end{tabular}

very differently than at higher aspect ratios. The proposed DNN model automatically learns the input-output dependencies and can serve as an accurate metamodel in point-particle simulations. The main advantage of DDN-based models is that the correlation procedure does not require a priori information about the dependency of an output variable on an input variable, a necessary condition for curve fitting methods. Furthermore, DNN models are applicable in cases of large parameter space without introducing unsatisfactory high biases.

We tested the performance of the constructed DNN model in a point-particle simulation of a settling problem. The agreement between the numerical and experimental data proved the capability of the DNN model in interpolating and extrapolating a learned relationship.

In this paper, we considered a single aspect ratio at $0 \leqslant$ $\mathrm{Re} \leqslant 120$. Data for other aspect ratio or Reynolds number ranges can be used as input in a straightforward manner to extend the model to other aspect ratios and Reynolds numbers. The DDN-based correlation procedure presented in this study can be used to predict other fluid-particle hydrodynamic interactions, such as velocity-gradient induced lift, and transient effects, such as history and added mass forces. Once a new database is generated, a model can be extended by just adding additional input neurons and training the network with the new data set.

\section{ACKNOWLEDGMENTS}

This work is part of the Research Programme "Innovative Magnetic Density Separation for the optimal use of resources and energy" under Project No. 14916, which is partly financed by the Netherlands Organisation for Scientific Research (NWO). The stay of Max Hausmann at Eindhoven University of Technology was funded by Erasmus+.

S.T. and M.H. contributed equally to this work. S.T. and M.H.: conceptualization, investigation, programming, postprocessing, validation, and writing-original draft. J.G.M.: experiments. J.G.M.K.: conceptualization, supervision, writing-review and editing. J.C.H.Z.: supervision, writing-review and editing. J.F.: supervision, writingreview and editing.

\section{APPENDIX}

The collected lumped data for steady drag, lift, and pitching torque coefficients are given in Tables III, IV, and V, respectively. The coefficients are based on the cross sectional area of the volume-equivalent sphere.
[1] R. Clift, J. R. Grace, and M. E. Weber, Bubbles, Drops, and Particles (Courier, New York, 2005).

[2] H. I. Andersson and A. Soldati, Anisotropic particles in turbulence: status and outlook, Acta Mech. 224, 2219 (2013).

[3] G. Bagheri and C. Bonadonna, On the drag of freely falling non-spherical particles, Powder Technol. 301, 526 (2016).

[4] G. A. Voth and A. Soldati, Anisotropic particles in turbulence, Annu. Rev. Fluid Mech. 49, 249 (2017).
[5] S. Elghobashi and G. C. Truesdell, Direct simulation of particle dispersion in a decaying isotropic turbulence, J. Fluid Mech. 242, 655 (1992).

[6] F. L. Yang and M. L. Hunt, Dynamics of particle-particle collisions in a viscous liquid, Phys. Fluids 18, 121506 (2006).

[7] J. K. Eaton, Two-way coupled turbulence simulations of gasparticle flows using point-particle tracking, Int. J. Multiphase Flow 35, 792 (2009). 
[8] J. G. M. Kuerten, Point-particle dns and les of particle-laden turbulent flow-a state-of-the-art review, Flow Turbul. Combust. 97, 689 (2016).

[9] A. B. Basset, A Treatise on Hydrodynamics: With Numerous Examples (Deighton, Bell, Cambridge, UK, 1888), Vol. 2.

[10] A. Oberbeck, Über stationäre Flüssigkeitsbewegungen mit Berücksichtigung der inneren Reibung, J. Reine. Angew. Math. 1876, 62 (1876).

[11] G. B. Jeffery, The motion of ellipsoidal particles immersed in a viscous fluid, Proc. R. Soc. London, Ser. A 102, 161 (1922).

[12] J. Happel and H. Brenner, Low Reynolds Number Hydrodynamics: With Special Applications to Particulate Media (Springer, Berlin, 2012), Vol. 1.

[13] F. G. Fan and G. Ahmadi, Dispersion of ellipsoidal particles in an isotropic pseudo-turbulent flow field, J. Fluids Eng. 117, 154 (1995).

[14] C. Marchioli, A. Fantoni, and M. Soldati, Orientation, distribution, and deposition of elongated, inertial fibers in turbulent channel flow, Phys. Fluids 22, 033301 (2010).

[15] H. Zhang, G. Ahmadi, F. G. Fan, and J. B. McLaughlin, Ellipsoidal particles transport and deposition in turbulent channel flows, Int. J. Multiphase Flow 27, 971 (2001).

[16] M. Abolhassantash, M. M. Tavakol, O. Abouali, M. Yaghoubi, and G. Ahmadi, Deposition fraction of ellipsoidal fibers in the human nasal cavity- influence of non-creeping formulation of hydrodynamic forces and torques, Int. J. Multiphase Flow 126, 103238 (2020).

[17] F. Jiang, L. Zhao, H. I. Andersson, K. Gustavsson, A. Pumir, and $\mathrm{B}$. Mehlig, Inertial torque on a small spheroid in a stationary uniform flow, Phys. Rev. Fluids 6, 024302 (2021).

[18] R. L. Pitter, H. R. Pruppacher, and A. E. Hamielec, A numerical study of viscous flow past a thin oblate spheroid at low and intermediate reynolds numbers, J. Atmos. Sci. 30, 125 (1973).

[19] G. H. Ganser, A rational approach to drag prediction of spherical and nonspherical particles, Powder Technol. 77, 143 (1993).

[20] L. Rosendahl, Using a multi-parameter particle shape description to predict the motion of non-spherical particle shapes in swirling flow, Appl. Math. Model. 24, 11 (2000).

[21] E. Loth, Drag of non-spherical solid particles of regular and irregular shape, Powder Technol. 182, 342 (2008).

[22] A. Hölzer and M. Sommerfeld, New simple correlation formula for the drag coefficient of non-spherical particles, Powder Technol. 184, 361 (2008).

[23] M. Hölzer, and A. Sommerfeld, Lattice Boltzmann simulations to determine drag, lift and torque acting on non-spherical particles, Comput. Fluids 38, 572 (2009).

[24] M. Zastawny, G. Mallouppas, F. Zhao, and B. Van Wachem, Derivation of drag and lift force and torque coefficients for non-spherical particles in flows, Int. J. Multiphase Flow 39, 227 (2012).

[25] F. Jiang, J. P. Gallardo, and H. I. Andersson, The laminar wake behind a 6: 1 prolate spheroid at 45 incidence angle, Phys. Fluids 26, 113602 (2014).

[26] R. Ouchene, M. Khalij, A. Tanière, and B. Arcen, Drag, lift and torque coefficients for ellipsoidal particles: From low to moderate particle Reynolds numbers, Comput. Fluids 113, 53 (2015).
[27] R. Ouchene, M. Khalij, B. Arcen, and A. Tanière, A new set of correlations of drag, lift and torque coefficients for non-spherical particles and large Reynolds numbers, Powder Technol. 303, 33 (2016).

[28] S. K. P. Sanjeevi and J. T. Padding, On the orientational dependence of drag experienced by spheroids, J. Fluid Mech. 820, 1 (2017).

[29] S. K. P. Sanjeevi, J. A. M. Kuipers, and J. T. Padding, Drag, lift and torque correlations for non-spherical particles from Stokes limit to high Reynolds numbers, Int. J. Multiphase Flow 106, 325 (2018).

[30] K. Fröhlich, M. Meinke, and W. Schröder, Correlations for inclined prolates based on highly resolved simulations, J. Fluid Mech. 901, A5 (2020).

[31] H. I. Andersson and F. Jiang, Forces and torques on a prolate spheroid: low-Reynolds-number and attack angle effects, Acta Mech. 230, 431 (2019).

[32] G. Akiki, T. L. Jackson, and S. Balachandar, Pairwise interaction extended point-particle model for a random array of monodisperse spheres, J. Fluid Mech. 813, 882 (2017).

[33] C. E. Brennen, Cavitation and Bubble Dynamics (Cambridge University Press, Cambridge, UK, 2014).

[34] G. Subramanian and D. L. Koch, Inertial effects on fibre motion in simple shear flow, J. Fluid Mech. 535, 383 (2005).

[35] S. L. Brunton, B. R. Noack, and P. Koumoutsakos, Machine learning for fluid mechanics, Annu. Rev. Fluid Mech. 52, 477 (2020).

[36] F. O. Sarghini, G. De Felice, and S. Santini, Neural networks based subgrid scale modeling in large eddy simulations, Comput. Fluids 32, 97 (2003).

[37] J. Ling, A. Kurzawski, and J. Templeton, Reynolds averaged turbulence modeling using deep neural networks with embedded invariance, J. Fluid Mech. 807, 155 (2016).

[38] L. He and D. K. Tafti, A supervised machine learning approach for predicting variable drag forces on spherical particles in suspension, Powder Technol. 345, 379 (2019).

[39] H. F. S. Lui and W. R. Wolf, Construction of reduced-order models for fluid flows using deep feedforward neural networks, J. Fluid Mech. 872, 963 (2019).

[40] M. Buzzicotti, F. Bonaccorso, P. C. Di Leoni, and L. Biferale, Reconstruction of turbulent data with deep generative models for semantic inpainting from turb-rot database, arXiv:2006.09179.

[41] I. Goodfellow, Y. Bengio, and A. Courville, Deep Learning (MIT Press, Cambridge, MA, 2016).

[42] J. H. Masliyah and N. Epstein, Numerical study of steady flow past spheroids, J. Fluid Mech. 44, 493 (1970).

[43] B. J. O'Donnell and B. Helenbrook, Drag on ellipsoids at finite reynolds numbers, At. Sprays 15, 363 (2005).

[44] F. W. Roos and W. W. Willmarth, Some experimental results on sphere and disk drag, AIAA J. 9, 285 (1971).

[45] M. Chrust, G. Bouchet, and J. Dušek, Numerical simulation of the dynamics of freely falling discs, Phys. Fluids 25, 044102 (2013).

[46] AB COMSOL, COMSOL Multiphysics Reference Manual, Version 5.3 (COMSOL AB, Stockholm, Sweden, 2018).

[47] M. Chrust, G. Bouchet, and J. Dušek, Parametric study of the transition in the wake of oblate spheroids and flat cylinders, J. Fluid Mech. 665, 199 (2010). 
[48] A. R. Shenoy and C. Kleinstreuer, Flow over a thin circular disk at low to moderate reynolds numbers, J. Fluid Mech. 605, 253 (2008).

[49] S. Ruder, An overview of gradient descent optimization algorithms, arXiv:1609.04747.

[50] X. Chen, S. Liu, R. Sun, and M. Hong, On the convergence of a class of adam-type algorithms for non-convex optimization, arXiv: 1808.02941.

[51] D. P. Kingma and J. Ba, Adam: A method for stochastic optimization, arXiv:1412.6980.

[52] M. Abadi, A. Agarwal, P. Barham, E. Brevdo, Z. Chen, C. Citro, G. S. Corrado, A. Davis, J. Dean, M. Devin, S. Ghemawat, I. Goodfellow, A. Harp, G. Irving, M. Isard, Y. Jia, R. Jozefowicz, L. Kaiser, M. Kudlur, J. Levenberg, D. Mané, R. Monga, S. Moore, D. Murray, C. Olah, M. Schuster, J. Shlens, B. Steiner, I. Sutskever, K. Talwar, P. Tucker, V. Vanhoucke, V. Vasudevan, F. Viégas, O. Vinyals, P. Warden, M. Wattenberg, M. Wicke, Y. Yu, and X. Zheng, TensorFlow: Large-scale machine learning on heterogeneous systems (2015), software available from tensorflow.org.

[53] See Supplemental Material at http://link.aps.org/supplemental/ 10.1103/PhysRevE.103.023304 for weights and biases corresponding to the DNN correlation for steady drag, lift, and pitching torque.
[54] W. W. Willmarth, N. E. Hawk, and R. L. Harvey, Steady and unsteady motions and wakes of freely falling disks, Phys. Fluids 7, 197 (1964).

[55] P. Ern, F. Risso, D. Fabre, and J. Magnaudet, Wake-induced oscillatory paths of bodies freely rising or falling in fluids, Annu. Rev. Fluid Mech. 44, 97 (2012).

[56] F. Auguste, J. Magnaudet, and D. Fabre, Falling styles of disks, J. Fluid Mech. 719, 388 (2013).

[57] S. Tajfirooz, J. G. Meijer, R. A. Dellaert, A. M. Meulenbroek, J. C. H. Zeegers, and J. G. M. Kuerten, Direct numerical simulation of magneto-archimedes separation of spherical particles, J. Fluid Mech. 910, A52 (2021).

[58] R. E. Rosensweig, Fluidmagnetic buoyancy, AIAA J. 4, 1751 (1966).

[59] Ronald E Rosensweig, Ferrohydrodynamics (Courier, New York, 2013).

[60] R. Y. S. Lai and L. F. Mockros, The stokes-flow drag on prolate and oblate spheroids during axial translatory accelerations, J. Fluid Mech. 52, 1 (1972).

[61] M. Van Hinsberg, J. H. M. ten Thije Boonkkamp, and H. J. H. Clercx, An efficient, second order method for the approximation of the basset history force, J. Comput. Phys. 230, 1465 (2011). 